Florida International University

FIU Digital Commons

4-11-2019

\title{
Best Probable Subset: A New Method for Reducing Data Dimensionality in Linear Regression
}

\author{
Elieser Nodarse \\ Florida International University, enoda006@fiu.edu
}

Follow this and additional works at: https://digitalcommons.fiu.edu/etd

Part of the Applied Statistics Commons, Multivariate Analysis Commons, Probability Commons, Social Statistics Commons, Statistical Methodology Commons, and the Statistical Models Commons

\section{Recommended Citation}

Nodarse, Elieser, "Best Probable Subset: A New Method for Reducing Data Dimensionality in Linear Regression" (2019). FIU Electronic Theses and Dissertations. 4280.

https://digitalcommons.fiu.edu/etd/4280

This work is brought to you for free and open access by the University Graduate School at FIU Digital Commons. It has been accepted for inclusion in FIU Electronic Theses and Dissertations by an authorized administrator of FIU Digital Commons. For more information, please contact dcc@fiu.edu. 


\section{FLORIDA INTERNATIONAL UNIVERSITY}

Miami, Florida

BEST PROBABLE SUBSET: A NEW METHOD FOR REDUCING DATA DIMENSIONALITY IN LINEAR REGRESSION

A thesis submitted in partial fulfillment of the

requirements for the degree of

MASTER OF SCIENCE

in

STATISTICS

by

Elieser Nodarse 
To: Dean Michael R. Heithaus

College of Arts, Sciences, and Education

This thesis, written by Elieser Nodarse, and entitled Best Probable Subset: A New Method for Reducing Data Dimensionality in Linear Regression, having been approved in respect to style and intellectual content, is referred to you for judgment.

We have read this thesis and recommend that it be approved.

Sneh Gulati

Golam Kibria

Zhenmin Chen

Hassan Zahedi, Major Professor

Date of Defense: April 11, 2019

The thesis of Elieser Nodarse is approved.

Dean Michael R. Heithaus

College of Arts, Sciences, and Education

Andrés G. Gil

Vice President for Research and Economic Development And Dean of the University Graduate School

Florida International University, 2019 


\section{DEDICATION}

I dedicate this thesis to my family: Maria T. del Cristo, Orlando Nodarse, Orlando Nodarse

Jr., Ernesto Carnota, and Shadow Nodarse. This thesis would not have been possible without your love and support. I love all of you, always. 


\section{ACKNOWLEDGMENTS}

I would like to thank my Major Professor, Dr. Hassan Zahedi, for guiding me throughout my professional career during my years at FIU. I would also like to thank the rest of my committee members and other professors who helped throughout the process of my thesis, Dr. Sneh Gulati, Dr. Golam Kibria, Dr. Zhenmin Chen, Dr. Florence George, and Professor Sergio Perez. Thank you for your guidance and support. Finally, I am grateful to the Department of Mathematics and Statistics and Florida International University for giving me the opportunity to study and excel in what I love. 


\title{
ABSTRACT OF THE THESIS \\ BEST PROBABLE SUBSET: A NEW METHOD FOR REDUCING DATA \\ DIMENSIONALITY IN LINEAR REGRESSION
}

\author{
by \\ Elieser Nodarse \\ Florida International University, 2019 \\ Miami, Florida, \\ Professor Hassan Zahedi, Major Professor
}

Regression is a statistical technique for modeling the relationship between a dependent variable $\mathrm{Y}$ and two or more predictor variables, also known as regressors [1]. In the broad field of regression, there exists a special case in which the relationship between the dependent variable and the regressor(s) is linear. This is known as linear regression.

The purpose of this paper is to create a useful method that effectively selects a subset of regressors when dealing with high dimensional data and/or collinearity in linear regression. As the name depicts, high dimensional data occurs when the number of predictor variables is far too large to use commonly known methods. Collinearity, on the other hand, occurs when there exists a linear relationship amongst one or more pairs of independent variables. This paper is divided into three main section: an introduction, which reviews key concepts that are needed for a full understanding of the paper; the methodology, which guides the reader, step-by-step, through the process of the newly devised method; results, which thoroughly explain and analyze any findings and propose further ideas to be studied. 


\section{TABLE OF CONTENTS}

CHAPTER

PAGE

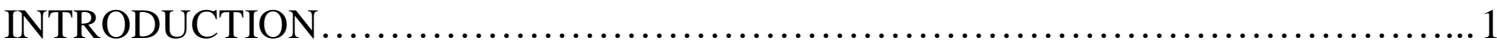

RESEARCH PURPOSE ................................................. 1

GENERAL INFORMATION ...........................................

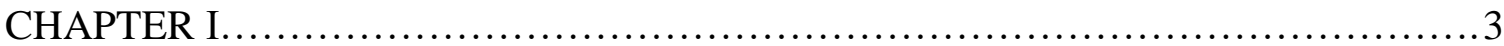

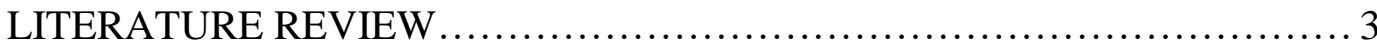

Regression......................................................

Least Squares Estimation........................................... 6

Violation of the LS Assumptions......................................

Quality of Fit of the Model.......................................... 10

High Dimensional Data............................................13

Subset Selection.................................................. 14

Regression Trees and Random Forest.................................19

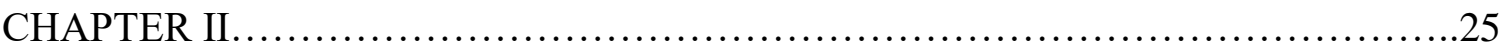

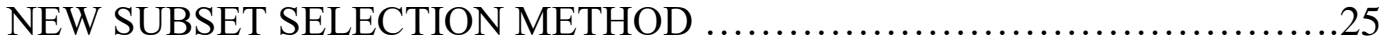

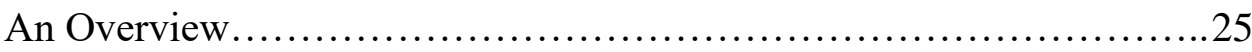

Step One: The Linear Relationship Pool................................ 29

Step Two: Selection of Predictor Variables................................32

Step Three: Choosing the Model with the Best $l$ Regressors.............. 35

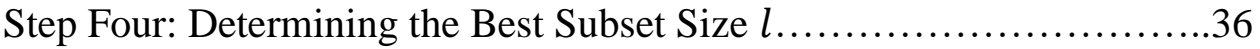

High Dimensional Data and Stopping Rules........................... 38

Best Probable Method Algorithm......................................40

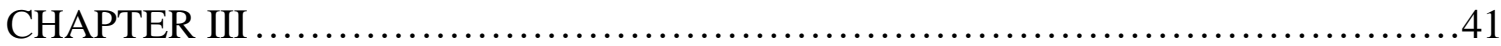

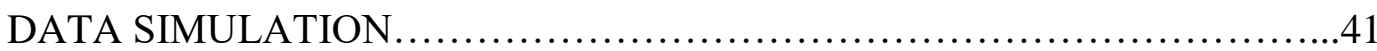

DATA COLLECTION.................................................. 43

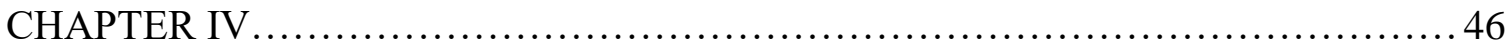

COMPARISONS AND RESULTS..........................................46

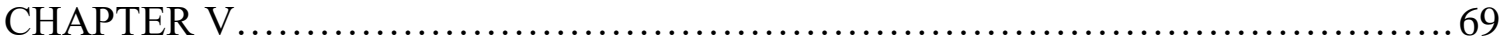

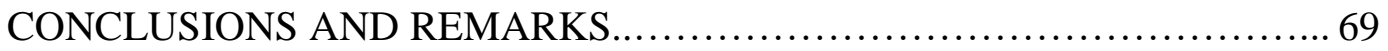

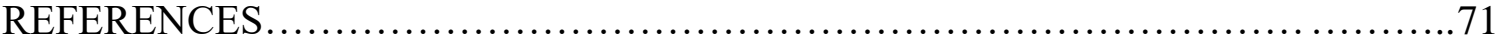

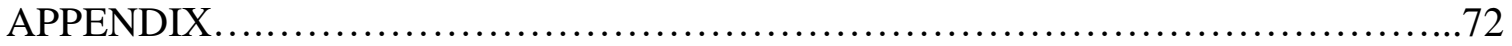




\section{LIST OF TABLES}

TABLE

PAGE

Table 1: Correlation Matrix - Dataset \# 1..................................... 47

Table 2: Regression Coefficients - Dataset \# 1................................47

Table 3: Best Probable Subset of Size $l$ - Dataset \# 1 ............................48

Table 4: Best Probable Subset LS Estimates - Dataset \# 1 ...........................49

Table 5: Subset Selection Methods $R_{a d j}^{2}$ Comparisons - Dataset \# 1...................49

Table 6: Random Forest Summary - Dataset \# 1 ................................50

Table 7: Regression Coefficients - Dataset \# 2 ...............................51

Table 8: Best Probable Subset of Size $l$ - Dataset \# 2 ..........................52

Table 9: Best Probable Subset LS Estimates - Dataset \# 2 ..........................52

Table 10: Subset Selection Methods $R_{a d j}^{2}$ Comparisons - Dataset \# 2 $\ldots \ldots \ldots \ldots \ldots \ldots . . .53$

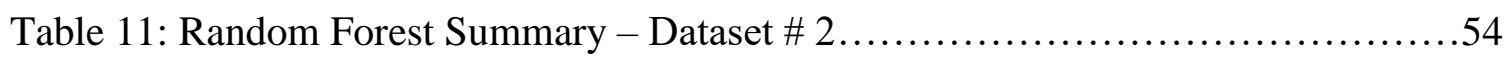

Table 12: Regression Coefficients - Dataset \# 3................................55

Table 13: Best Probable Subset of Size $l$ - Dataset \# 3..........................56

Table 14: Best Probable Subset LS Estimates - Dataset \# 3.........................57

Table 15: Subset Selection Methods $R_{a d j}^{2}$ Comparisons - Dataset \# 3.................58

Table 16: Subset Selection Methods $R^{2}$ Comparisons - Dataset \# 3 ....................59

Table 17: Random Forest Summary - Dataset \# 3 ...............................59

Table 18: Regression Coefficients - Dataset \# 4 ...............................60

Table 19: Best Probable Subset of Size $l$ - Dataset \# 4 ..........................62

Table 20: Best Probable Subset LS Estimates - Dataset \# 4 ........................6 62

Table 21: Subset Selection Methods $R_{a d j}^{2}$ Comparisons - Dataset \# 4................63

Table 22: Random Forest Summary - Dataset \# 4............................... 63

Table 23: Best Probable Subset of Size $l$ - Dataset \# 5...........................66

Table 24: Regressor Numbers and Names - Dataset \# 5 ........................66

Table 25: Best Probable Subset LS Estimates - Dataset \# 5........................67 
Table 26: Subset Selection Methods $R_{a d j}^{2}$ Comparisons - Dataset \# 5................68

Table 27: Random Forest Summary - Dataset \# 5.............................68 


\section{LIST OF FIGURES}

FIGURE

PAGE

Figure 1: Schematic for Statistical Models...................................... 3

Figure 2: A visual representation of a Linear Model...............................5

Figure 3: Linearity assumption not met...................................... 8

Figure 4: Constant-Variance assumption not met................................ 9

Figure 5: Splitting of a regressor space (left) and the creation of regions in the

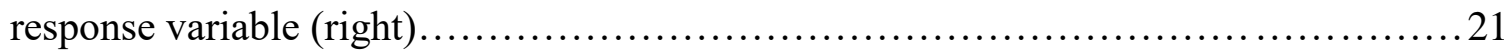

Figure 6: A visual of a Random Forest using two trees............................23

Figure 7: Diagram of the Best Probable Subset (BPS) method......................28

Figure 8: Step One: Creating the Linear Relationship Pool (LRP).................... 29

Figure 9: Step One: Alternative sampling plan................................. 30

Figure 10: Step Two: Selecting a subset of $l$ regressors using the preferred and alternative sampling plans and a comparison between the two....................... 33

Figure 11: Step Three: Selecting the model with the best subset of size $l \ldots \ldots \ldots \ldots \ldots . \ldots 35$

Figure 12: Adjusted R-Squares vs. Subset Size Plot - Dataset \# 1................... 48

Figure 13: Adjusted R-Squares vs. Subset Size Plot - Dataset \# 2 ................... 51

Figure 14: Adjusted R-Squares vs. Subset Size Plot - Dataset \# 3...................56

Figure 15: Adjusted R-Squares vs. Subset Size Plot - Dataset \# 4 ................... 61

Figure 16: Residuals vs. Fitted Values - Dataset \# 5............................. 64

Figure 17: Adjusted R-Squares vs. Subset Size Plot - Dataset \# 5 ................... 65 


\section{INTRODUCTION}

\section{RESEARCH PURPOSE}

The principal purpose of this paper is to present a new method that deals with high dimensional data and/or collinearity in linear regression.

\section{GENERAL INFORMATION}

Regression Analysis is a statistical technique used for modeling and analyzing the relationship between a response variable $Y$ and one or more predictor variables $X_{1}$, $X_{2}, \ldots, X_{k}[1]$. When the relationship between $Y$ and each of the predictors $X_{1}, X_{2}, \ldots, X_{k}$ is known or assumed to be linear, we use a linear approach, commonly known as Linear Regression. Some of the main purposes of linear regression analysis include data collection as well as fitting and estimating the regression parameters of a linear regression model, which can provide us with useful information about the relationship between the response variable and one or more predictor variables. Data collection is very essential, as "any regression analysis is only as good as the data on which it is based" [1].

A very popular method for estimating the regression parameters of a linear model is known as Least Squares Estimation (LSE) [2]. However, the LSE method cannot be used when the number of regressors equals or exceeds the number of observations in the data, a phenomenon known as high dimensionality [3]. Using the LSE can overfit the data when the number of predictors equals the number of observations, which means that the data will follow the error too closely. There are some existing solutions to data having high dimensionality, including Principal Component Analysis, which transforms the data, deriving a low-dimensional set of orthogonal variables [3]. 
In the present paper we focus on methods for reducing the dimensions of data sets without having to transform the data set. Most of the methods that we will discuss belong to the Subset Selection class, which select a smaller subset of regressors [3]. After thoroughly exploring these methods, we define and propose a new subset selection method, which we call Best Probable Subset (BPS) in hopes of fitting linear regression models containing only those predictor variables that contribute to the model while keeping unnecessary variables out of the model. 


\section{CHAPTER I}

\section{LITERATURE REVIEW}

A Statistical Model is a quantity in which we relate a set of input variables to an output variable, along with some variability [4] (Figure 1). We begin this paper by reviewing a specific type of statistical models -

Linear Regression model - and

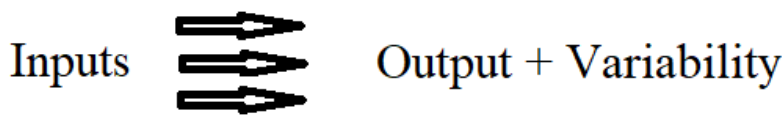
reviewing some of the most widely

Figure 1: Schematic for Statistical Models. (Reproduced and used techniques to estimate and updated from Methods and Applications of Linear Models, Hocking, $2^{\text {nd }} e d$.)

analyze these models.

\section{Regression}

Regression is a statistical technique for modeling the relationship between a response sometimes called dependent- variable $Y$ and one or more predictor variables, or regressors. Regression Analysis deals with finding and modeling the best relationship between $Y$ and the regressor(s), quantifying the strength of said relationship, and predicting future response values for a given set of values of the regressors [6]. Amongst all regression models, a special case exists in which the relationship between the dependent variable $Y$ and the regressors is linear; this is known as Linear Regression [1]. Linear regression can be further divided into two commonly known cases. The first case involves a response variable $Y$ and only one predictor variable $X$. This is known as Simple Linear Regression [1] and it has the general model:

$$
Y=\beta_{0}+\beta X+\varepsilon
$$

where $\beta_{0}$ and $\beta$ are unknown parameters known as regression coefficients. Specifically, $\beta_{0}$ is known as the $Y$-Intercept -the value that $Y$ assumes when $X$ is zero, if it can be zero- 
and $\beta$ is the slope, which determines how $Y$ changes with every unit change in $X$ [1]. One of the purposes of linear regression analysis is to estimate those parameters. Lastly, $\varepsilon$ (epsilon), distributed as $\left(0, \sigma^{2}\right)$, is a random variable that accounts for the error, or difference, between the linear function of $X-\beta_{0}+\beta X$ - and the observed values of the variable $Y$ [1]. Also, it is assumed that $\sigma^{2}$ is unknown [5]. Here we can see that linear regression models can be viewed as having a predictable part, $\beta_{0}+\beta X$, and an unpredictable part, $\varepsilon$ [7]. There are some assumptions that must be met for us to perform any linear regression analysis on the regression model. These assumptions are formally listed here for the convenience of the reader:

1. The relationship between the predictor variable(s) and the response variable must be linear [7].

2. The error term $\varepsilon$ is distributed as $N\left(0, \sigma^{2}\right)[7]$

3. The errors of the response variable are independent of each other [7]

4. Predictor variables are independent of each other (i.e. no collinearity) [7]

For each value of $x$, there is a population of possible values, $Y \mid x$, for the response variable, which follows the model:

$$
Y \mid x=\beta_{0}+\beta x+\varepsilon
$$

We use the notation $Y \mid x$ to indicate the population of values for the variable $Y$ given a value of $x$, and $y$ to indicate an observed value of $Y \mid x$. Figure 2 shows a visual representation of the model $Y \mid x=\beta_{0}+\beta x+\varepsilon$. Note that there is a distribution for the population of possible values in $Y \mid x$, when $X$ assumes a value $x$. The $Y \mid x$ population has a mean equal to the linear model at a given value of $x$ and a constant variance $\sigma^{2}$. This occurs 
because the value $x$ is fixed and $\varepsilon \sim N\left(0, \sigma^{2}\right)$, so the mean of $Y \mid x$ is a linear function of the $x[2]$, and the variance equals the variance of $\varepsilon$ :

$$
E(Y \mid x)=\beta_{0}+\beta x \text { and } V(Y \mid x)=\sigma^{2}
$$

The other case of linear regression involves a response variable and two or more predictor variables, which is known as Multiple Linear Regression [1] and the multiple linear regression model follows the format:

$$
Y=\beta_{0}+\beta_{1} X_{1}+\beta_{2} X_{2}+\cdots+\beta_{k} X_{k}+\varepsilon
$$

In (3), $Y$ is the response variable while $X_{1}, X_{2}, \ldots, X_{k}$ are the predictor variables. Parameter $\beta_{0}$ is known as the $Y$ intercept -the value of $Y$ when all regressors are assumed to have a

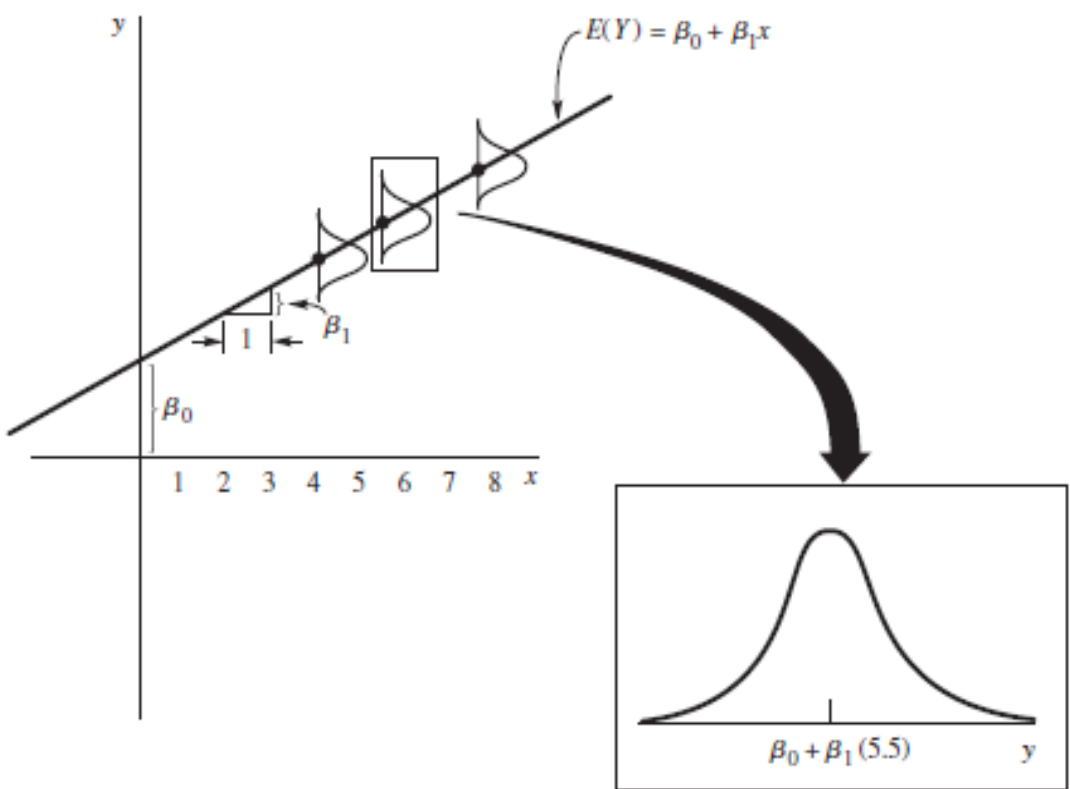
value of zero, if it makes sense for them

Figure 2: A visual representation of a Linear Model. (Figure taken from Mathematical Statistics with Applications, Wackerly, $7^{\text {th }}$ ed.)

to be zero- and parameters $\beta_{1}, \beta_{2}, \ldots, \beta_{k}$ are the regression coefficients, which determine how regressors $X_{1}, X_{2}, \ldots, X_{k}$, respectively, influence $Y$. Like simple linear regression, $\varepsilon$ is the random variable accounting for the difference between the model and the observed values of the variable $Y$, and it is distributed as $N\left(0, \sigma^{2}\right)$ [1]. In equation (3), the response variable depends on variables $X_{1}, X_{2}, \ldots, X_{k}$. Similar to simple linear regression, for a set 
of fixed values $x_{1}, x_{2}, \ldots, x_{k}$, the response variable $Y \mid x_{1}, x_{2}, \ldots, x_{k}$ has a population of possible values. Essentially, the response variable $Y \mid x_{1}, x_{2}, \ldots, x_{k}$ follows the model:

$$
Y \mid x_{1}, x_{2}, \ldots, x_{k}=\beta_{0}+\beta_{1} x_{1}+\beta_{2} x_{2}+\cdots+\beta_{k} x_{k}+\varepsilon
$$

Since the set of values $x_{1}, x_{2}, \ldots, x_{k}$ are fixed and $\varepsilon \sim N\left(0, \sigma^{2}\right)$, the mean of $Y \mid x_{1}, x_{2}, \ldots, x_{k}$ is a linear function of the regressors, and the variance equals the variance of $\varepsilon$ [1]. In other words:

$$
E\left(Y \mid x_{1}, x_{2}, \ldots, x_{k}\right)=\beta_{0}+\beta_{1} x_{1}+\beta_{2} x_{2}+\cdots+\beta_{k} x_{k} \text { and } V\left(Y \mid x_{1}, x_{2}, \ldots, x_{k}\right)=\sigma^{2}
$$

In order to model the relationship between the response variable $Y$ and the predictor variable(s), we must first estimate the regression parameters $\beta_{0}, \beta_{1}, \ldots, \beta_{k}$, which brings us to the next section.

\section{Least Squares Estimation}

The Method of Least Squares (LS) is used to estimate the regression coefficients in both simple and multiple linear regression. For simplicity purposes, let us consider this method in simple linear regression first and then in multiple linear regression.

Suppose we have $n$ pairs of data $\left(x_{1}, y_{1}\right),\left(x_{2}, y_{2}\right), \ldots,\left(x_{n}, y_{n}\right)$. Note that here we only have one predictor variable and one response variable. The subscripts from 1 to $n$ simply define the different data sets. The Method of Least Squares estimates the parameters $\beta_{0}$ and $\beta_{1}$ and generates a fitted linear regression model $\hat{Y}=b_{0}+b_{1} x$, where $b_{0}$ and $b_{1}$ are unbiased Least Squares Estimators (LSE) of $\beta_{0}$ and $\beta_{1}$, respectively, making $\hat{Y}$ an unbiased estimator of the model, so $E(Y \mid x)=\beta_{0}+\beta x$ [2]. For each given value $x_{1}$,

$x_{2}, \ldots, x_{n}$, the fitted model generates a corresponding fitted value $\hat{y}_{1}, \hat{y}_{2}, \ldots, \hat{y}_{n}[1]$. Without diving into the formulas and computations used by the method of least squares, we only 
need to know that said method provides us with estimates that minimize the sum of squares of the difference between the observations $y_{1}, y_{2}, \ldots, y_{n}$ and the fitted values $\hat{y}_{1}, \hat{y}_{2}, \ldots, \hat{y}_{n}$ [1]. Let us note here that the difference between an observed value $y_{i}$ and a fitted value $\hat{y}_{i}$, both corresponding to the same $\mathrm{x}_{1}$, is known as a residual $\mathrm{e}_{i}$ [3].

The LS method follows the same idea for multiple linear regression: It provides us with estimates for $\beta_{1}, \beta_{2}, \ldots, \beta_{k}$ which minimize the sum of squares of the residuals $\mathrm{e}_{i}=\mathrm{y}_{i}-$ $\hat{\mathrm{y}}_{i}$, for $1 \leq i \leq n[1]$. For the purpose of the current paper, we will review the estimates of the regression parameters in matrix form. Suppose that we have $n$ observations on a response variable $Y \mid x$ and $k$ predictor variables:

$$
\left(x_{11}, x_{12}, \ldots, x_{1 k}, y_{1}\right),\left(x_{21}, x_{22}, \ldots, x_{2 k}, y_{2}\right), \ldots,\left(x_{n 1}, x_{n 2}, \ldots, x_{n k}, y_{n}\right)
$$

We define the following matrices:

$$
\boldsymbol{y}=\left[\begin{array}{c}
y_{1} \\
y_{2} \\
\vdots \\
y_{n}
\end{array}\right], \boldsymbol{X}=\left[\begin{array}{ccccc}
1 & x_{11} & x_{12} & \ldots & x_{1 k} \\
1 & x_{21} & x_{22} & \ldots & x_{2 k} \\
\vdots & \vdots & \vdots & \ddots & \vdots \\
1 & x_{n 1} & x_{n 2} & \ldots & x_{n k}
\end{array}\right], \boldsymbol{\beta}=\left[\begin{array}{c}
\beta_{0} \\
\beta_{1} \\
\vdots \\
\beta_{k}
\end{array}\right], \boldsymbol{\varepsilon}=\left[\begin{array}{c}
\varepsilon_{1} \\
\varepsilon_{2} \\
\vdots \\
\varepsilon_{n}
\end{array}\right]
$$

The solution for finding the estimates of the correlation coefficients is given by:

$$
\boldsymbol{b}=\left(\boldsymbol{X}^{T} \boldsymbol{X}\right)^{-1} \boldsymbol{X}^{T} \boldsymbol{y}
$$

The proof and specifics for obtaining the least squares estimates $\boldsymbol{b}$ are covered in Douglas C. Montgomery's Introduction to Linear Regression Analysis or any Regression book cited in this paper.

\section{Violation of the LS Assumptions}

The Least Squares estimates $\boldsymbol{b}$ will have low bias provided that the true relationship between the predictor variable(s) and the response variable $Y$ is linear. Furthermore, if the number of observations $n$ is much larger than the number of regressors $k$, the least squares 
estimates tend to have a low variance [3]. In fact, if this and the rest of the assumptions presented earlier in the previous section are met, the Least Squares estimator is known to have the minimum variance amongst all unbiased estimators. Such estimators are known as the Minimum Variance Unbiased Estimators (MVUE) [2]. However, these assumptions are not always met and can bring along many problems. Here are some of the violated assumptions along with their respective outcomes:

\section{Non-linearity of the Data}

When the data are not from linear, the conclusions that we draw from the fitted model are suspect for inaccuracy. A good way to check if this assumption is met is to plot a Residuals vs. Fitted values plot. If a pattern is visible, the data might not be linear. Figure 3 shows an example of a non-linear dataset.

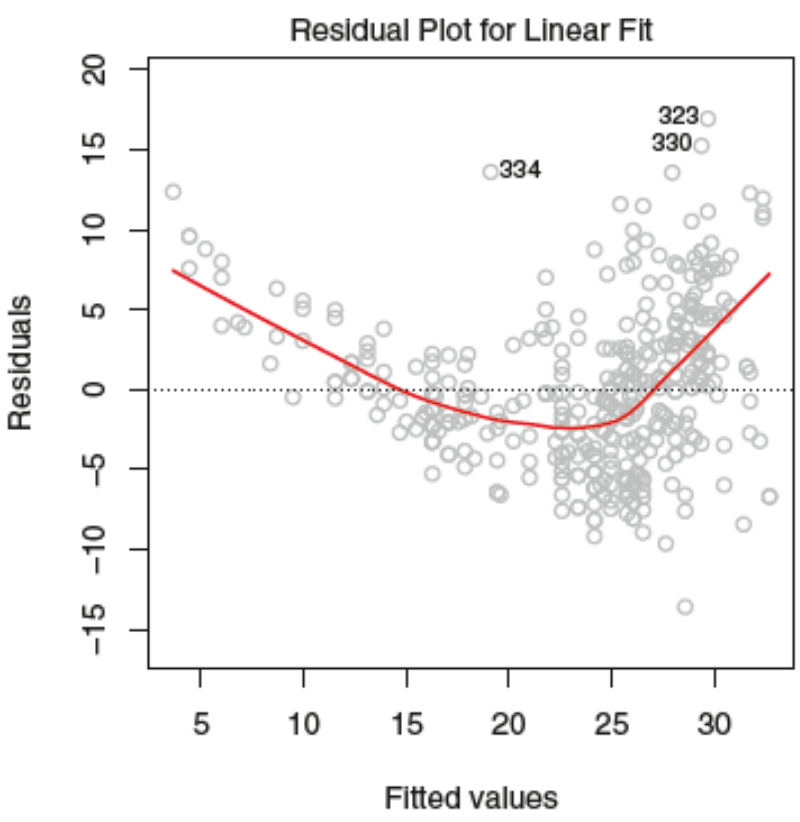

When this assumption is violated, a from Gareth James'An Introduction to Statistical Learning with Applications in R.)

simple transformation - such as

$\sqrt{X}, \log (X)$, or $X^{2}-$ could be appropriate for fitting a model [3].

\section{Correlation of Error Terms}

One of the assumptions to linear regression analysis is that the error terms of different observations are uncorrelated to each other. If this assumption is not met, the estimated 
standard errors of the estimated regression coefficients will underestimate the true standard errors, which will affect any confidence and prediction intervals that are constructed [3].

\section{Non-constant Variance of Error Terms}

The assumption that we have a constant variance for all error terms is important when making confidence intervals and hypothesis tests. If the assumption of equal variance is violated, such intervals and tests might be inaccurate. We can check this assumption by plotting the Residuals vs. Fitted values and keeping an eye for any

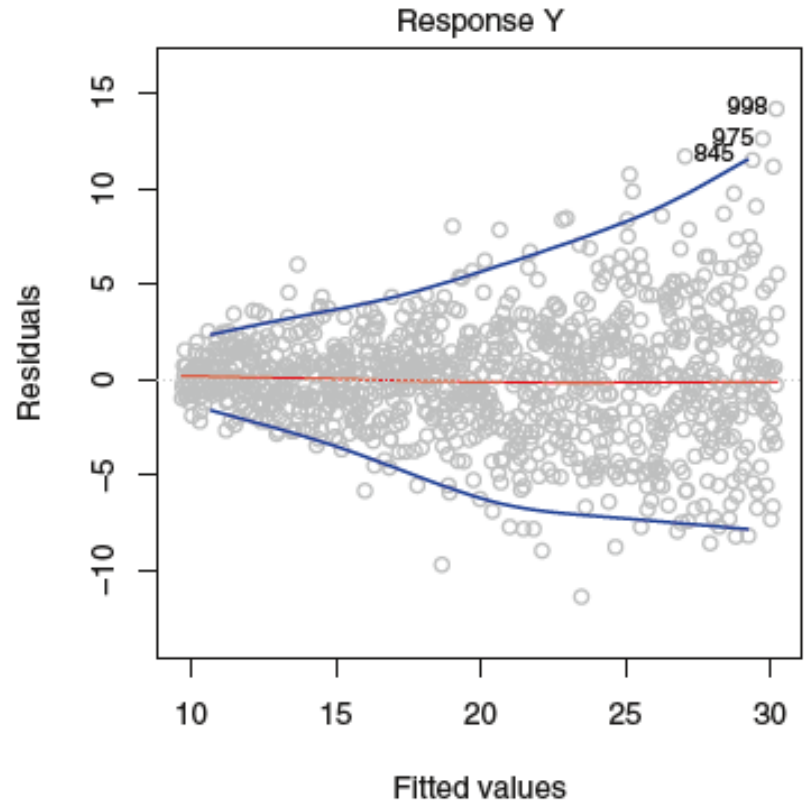

Figure 4: Constant-variance assumption not met. (Image taken from Gareth James' An Introduction to Statistical Learning with Applications in R.)

changes in the sparsity of the

Residuals vs Fitted Values plot. Figure 4 shows an example of a dataset in which the variance $\sigma^{2}$ is not constant. The non-constant variance problem can usually be solved by applying a transformation to the response variable $Y$, such as $\log (Y)$ or $\sqrt{Y}$ [3].

\section{Collinearity}

A measure of "closeness" or dependency between two variables, whether dependent or independent, is known as the correlation between those variables. Collinearity occurs when two predictor variables are closely related to each other [3]. The phenomenon of collinearity reduces the accuracy of the estimates of the regression coefficients and 
increases their standard error. Consequently, the t-statistics used for testing each individual coefficient is also reduced and the power of the hypothesis tests declines. Collinearity can be detrimental when fitting a model. Some ways to detect it include looking at the correlation matrix of all regressors or computing the variance inflation factor (VIF) [3]. When looking at the VIFs, we can drop one of the regressors that yield the highest VIF and check if the collinearity has been reduced or eliminated [4]. The details of VIFs are beyond the scope of this paper, but more information can certainly be found in Hocking's Methods and Applications of Linear Models, $3^{\text {rd }}$ Ed.

\section{Quality of Fit of the Model}

We have explored the Method of Least Squares when estimating the regression coefficients of a linear model. However, we cannot fit a regression model and hope that it will be a good estimate of the regression parameters. We need a way to measure how well the fitted values do at predicting the observed data. We present some statistics used for measuring the quality of fit of a fitted mode.

\section{Mean Squared Error}

The most commonly used measure of fit is known as Mean Squared Error (MSE) [3]. Like the name indicates, the MSE measures the average of the square of the errors between the observed data and the predicted values of that same data. The formula for the MSE is provided here:

$$
\operatorname{MSE}=\frac{1}{n} \sum_{i=1}^{n}\left(y_{i}-\hat{y}_{i}\right)^{2}
$$


where $y_{i}$ is the $i^{\text {th }}$ observation of the data containing $n$ observations and $\hat{\mathrm{y}}_{i}$ is the corresponding $i^{\text {th }}$ predicted value. Logically, we expect the value of MSE to be large if the predicted values are far off the observed ones. The quantity $\sum_{i=1}^{n}\left(y_{i}-\hat{y}_{i}\right)^{2}$, without the

term $\frac{1}{n}$ being multiplied by it, is known as the Sum of Squares of the Errors (SSE). The SSE quantity is a measure of the variability in $y$ after the predictor variable(s) have been considered [1].

\section{Coefficient of Determination: $R$-Squared}

The quantity $R$-Squares $\left(\mathrm{R}^{2}\right)$ is known as the coefficient of determination and it is a measure of the variability of the response variable $y$ that has been explained by the predictor variable(s) [1]. When we have a simple regression model - only one predictor variable - the $R^{2}$ is known as the squared of the correlation between the predictor variable and the response variable [3]. The measure of variability can be obtained using the following equation:

$$
R^{2}=\frac{S S R}{S S T}=\frac{\sum_{i=1}^{n}\left(\widehat{\mathrm{y}}_{i}-\overline{\mathrm{y}}\right)^{2}}{\sum_{i=1}^{n}\left(y_{i}-\overline{\mathrm{y}}\right)^{2}}
$$

where SSR is known as the Sum of Squares of Regression (SSR) and SST is the Total Sum of Squares (TSS). While SSE is the measure of variability in $y$ after the regressors have been considered, RSS is the variability of $y$ that is explained by the regressors. These two measurements make up the total variability in $y$, known as TSS. Naturally, these three measures make up the equation:

$$
T S S=S S R+S S E
$$


While MSE and R-Squared are both measures of variability in $y$, they offer different information. A low value in the MSE would be an indication of a good fitted model while a large value would indicate a bad fit. On the other hand, a small value of $R^{2}$ indicated a model with poor fit while a large value indicated a good fit [3]. This is because MSE measures the variability in the model not explained by the regressors while $R^{2}$ explains the variability explained by the regressors. Naturally, if we add one more predictor variable to a model, the MSE will decrease while the $R^{2}$ will decrease, whether slightly or considerably. As a result of the added predictor variable, it is best to use these two measurements of variability when comparing fitted models with the same number of regressors.

So which one should we use? This question cannot be answered directly, but we recommend using the $R^{2}$. While the MSE can be as large as we can imagine, $R^{2}$ is a fraction that can only take on values between 0 and 1 , where a value close to 1 indicates that the fitted model explains a large portion of the variability in the response variable [1]. This allows us to understand how good a fitted model is in general.

\section{Adjusted R-Squared}

As mentioned above, the value of $R^{2}$ of an already existing fitted model can only increase with the addition of another predictor variable while the MSE can only decrease. However, having a larger $R^{2}$ when the model has an extra regressor does not mean that one model is better with such inclusion. In fact, it could be better with or without it. So how do we know if the inclusion of another regressor improves the model? This question can be answered using other measurements. Particularly, we focus on a statistic known as the Adjust $R$ - 
Squared $\left(R_{a d j}^{2}\right)$ which is a modified version of $R^{2}$ that pays a price for the addition of unnecessary regressors in the model [3]. The formula for finding the adjusted R-Squared is provided here:

$$
R_{a d j}^{2}=1-\frac{\frac{S S E}{(n-k-1)}}{\frac{T S S}{(n-1)}}=1-\frac{\left(1-R^{2}\right)(n-1)}{(n-k-1)}
$$

where $k$ is the number of regressors in the model. Like the $R^{2}$ statistic, $R^{2}{ }_{a d j}$ can only take on values less than 1 [3]. However, unlike the non-adjusted version, $R^{2}{ }_{a d j}$ can decrease beyond zero with the addition of unnecessary predictor variables. The adjustment provides us with a tool to compare fitted models involving different numbers of regressors. While there are many more statistics used for comparing fitted models, they are beyond the scope of the present paper and we only focus on the ones mentioned above.

\section{High-Dimensional Data}

We mentioned earlier that the Least Squares Estimate is known to be the MVUE if all the assumptions are met. However, we may not be able to always find the LSE for all data sets, as it requires the number of observations $n$ to be much larger than the number of predictor variables $k$. A problem occurs when $n$ is not much larger than $k$. If $n$ is only slightly larger than $k$, there can be too much variability in the least squares estimates, leading to possibly overfitting the data and poorly predicting future observations [3]. What is worse, if $k$ is larger than $n$, the variances of the estimates are infinite, and the least squares method can no longer be used [3]. Data that fall under any one of these two scenarios are known to have high dimensionality. 
Multiple approaches exist when dealing with high dimensional data. Subset selection and Shrinkage are two common classes of methods used when dealing with high dimensionality [3]. Subset selection involves identifying a subset of the $k$ predictor variables that are related to the response $Y$. Once the subset is identified, we fit a reduced model using the Method of Least Squares [8]. Shrinkage, on the other hand, involves using all $k$ original predictors. The $k$ estimated regression coefficients are "shrunken" towards zero, relative to the LSE. Some methods of shrinkage include Ridge Regression and the Lasso method [3].

While we focus on subset selection as a primary class of methods for dimension reduction in the present paper, more information on the shrinkage class can be found in Montgomery's Introduction to Linear Regression Analysis, $5^{\text {th }}$ Ed. and Rawlings' Applied Regression Analysis: A Research Tool, $2^{\text {nd }}$ Ed. Another class approach used when dealing with high dimensional data involves Tree-Based methods. Tree-based methods are not as common as the other two mentioned above. However, we consider one method called Random Forest, which takes on a very different approach by selecting a subset of regressors for each split, or node, that occurs in any of the trees. We go more in depth into the subset selection class and the random forest method in the next few sections.

\section{Subset Selection}

Inside the Subset Selection class there are some useful methods that are commonly used when reducing the dimensions of a dataset. Here we present a summary and the algorithm for some of these methods. 


\section{Best Subset Selection}

Best subset selection can guarantee that we will find the best possible combination of the $k$ predictor variables [3]. The disadvantage of the best subset selection method is that we would have to fit a LS regression model for each of the $2^{k}$ possible combinations of the $k$ predictors, whether the model contains only one predictor or the original $k$ [3]. Best subset selection method can be very useful when the number of regressors is very small, so that we only have a small number of possible fitted models. However, it can be computationally inefficient. When the number of possible regressors is 10 , we already have $10^{2}=100$ fitted models to consider; when there are 32 possible regressors, there are over 1000 possible fitted models to consider. The algorithm for this method can be found in from Gareth James' An Introduction to Statistical Learning with Applications in $R$, $7^{\text {th }}$ Printing, but we summarize and present it here for your convenience:

1. Let $M_{0}$ represent the null model with no predictors and $M_{1}, M_{2}, \ldots, M_{k}$ represent models with $1,2, \ldots$, and $k$ predictor variables.

2. For $l=1,2, \ldots, k$, fit all possible $\left(\begin{array}{l}k \\ l\end{array}\right)$ models that contain exactly $l$ regressors. Pick the best model among each group of $\left(\begin{array}{l}k \\ l\end{array}\right)$ and call it $M_{l}$.

Note: The "best" model amongst all models with the same number of predictors $l$ is the one having the largest $R^{2}$.

3. Select the single best model among the $k+1$ models $M_{0}, M_{1}, M_{2}, \ldots, M_{k}$ using any of the $C_{p}(A I C), B I C$, or adjusted $R^{2}$ statistics. ${ }^{[3]}$

Note: The adjusted $R^{2}$ statistic is explained in a previous section. 


\section{Forward Stepwise Selection}

Forward Stepwise Selection is a computationally efficient method compared to subset selection as it does not involve checking every possible fitted model. Rather, the method

only considers a maximum of $\frac{k(k+1)}{2}+1$ possible fitted models, where $k$ is the number of regressors in the full model. A major disadvantage of Forward stepwise selection is that it does not consider the possibility that a predictor variable that has already been selected for the fitted model might no longer be significant with the addition of another predictor, so it might not yield the single best possible fitted model.

The method starts with the null model $M_{0}$ and considers the regressor that best contributes to the model, if there is any that contributes to it in a significant way. A "significant" addition is one that yields a partial $F$ statistic greater than a pre-selected value $F_{I N}$ for "taking in" a new regressor [7]. A review on the $F$ distribution can be found in Lyman Ott's An Introduction to Statistical Methods and Data Analysis, $7^{\text {th }}$ Ed. or any other referenced book that covers elementary Statistics. However, the textbook Introduction to Statistical Learning with Applications in $R$, used as a reference in this paper, presents an algorithm that avoids the calculation of the partial F-statistic. The Forward selection algorithm is summarized here:

1. Begin by fitting the null model, $M_{0}$.

2. For $l=0,1, \ldots, k-1$, consider every $k-l$ fitted model that adds a single predictor variable not already present in $M_{l}$. Choose the model that yields the highest $R^{2}$ and call it $M_{l+1}$. 
3. Select the single best model among the $M_{0}, M_{1}, M_{2}, \ldots, M_{k}$ possible models using any of the $C_{p}(A I C), B I C$, or adjusted $R^{2}$ statistics [3].

\section{Backward Stepwise Elimination}

Backward Elimination also has the computational advantage over Best Subset Selection, as it considers a maximum of $\frac{k(k+1)}{2}+1$ possible fitted models [3].

We begin the procedure with the full model, $M_{k}$, instead of the null model, $M_{0}$. We then eliminate the least significant regressor in the model, if there is any that is not statistically significant according to a criteria, which is whether the partial F-statistic is smaller than the cut-off point $F_{\text {OUT }}$ for "kicking out" an already present regressor [8]. For simplicity purposes, we summarize an algorithm here that does not involve the partial F-statistic. Like the previous subset selection methods, the Backward elimination algorithm was taken from Introduction to Statistical Learning with Applications in $R$ and summarized here:

1. Begin by fitting the full model, $M_{k}$.

2. For $l=k, k-1, \ldots, 1$, consider every of the $l$ possible fitted model that has one less predictor variable than $M_{l}$. Choose the model that yields the highest $R^{2}$ and call it $M_{l-1}$.

3. Select the single best model among the $M_{0}, M_{1}, M_{2}, \ldots, M_{k}$ possible models using any of the $C_{p}(A I C), B I C$, or adjusted $R^{2}$ statistics [3].

\section{Stepwise Regression}

Stepwise Regression combines both forward selection and backward elimination to create a procedure that keeps the computational advantages of these two methods while mimicking a close approach to the best subset selection method. In this procedure, we have 
two cut-off F-statistics, $F_{I N}$ and $F_{O U T}$, for "taking in" a new regressor into the model or "kicking out" an already present regressor, respectively. The value of $F_{I N}$ is typically chosen to be larger than $F_{\text {OUT }}$ so that it is more difficult to add a new regressor to the model than to delete one [1]. An algorithm for the stepwise regression method is not provided in Introduction to Statistical Learning with Applications in R. However, we still summarize the procedure here, as it is explained in An Introduction to Statistical Methods and Data Analysis, $7^{\text {th }} \mathrm{Ed}$.

1. Begin by fitting the null model, $M_{0}$, with no predictor variables.

2. Add to the model the predictor that yields the largest simple correlation with the response variable $y$.

3. For $l=1, \ldots, k-1$ :

a. Consider every $k-l$ fitted model that adds a single predictor variable not already in the $M_{l}$ model. If any of the $k-l$ models that have a partial $F$ statistic greater than a pre-selected value $F_{I N}$, select the one that has the highest partial $F$ statistic and name it $M_{l+1}$. If none of the models have a partial $F$ statistic exceeding $F_{I N}$, then $M_{l}$ is the final model.

b. Once the $M_{l+1}$ model is obtained, check if any of the predictors already in the model yield a partial $F$ statistic lower than $F_{\text {OUT }}$. If so, remove the predictor with the lowest partial $F$ statistic. Continue repeating this process until all irrelevant predictor variables have been removed.

c. Repeat steps (a) and (b) until no more regressors are added or removed [8]. 


\section{Disadvantages of Subset Selection Methods in High Dimensional Data}

The subset selection methods mentioned above have their own advantages and disadvantages when dealing with low-dimensional data. But what if the number of observations $n$ is larger than the number of predictor variables $k$ ? Best subset selection considers all possible models, so it cannot be used in high dimensional data because some models would be overfitting the data or the variances of the estimates would be infinite. Backward elimination starts with all regressors in the model, so it cannot be used in high dimensional data either. Forward selection, on the other hand, starts with the null model, so we can add predictor variables into the fitted model while $n$ is greater than $k$. However, as mentioned previously, forward selection has the disadvantages of keeping previously added regressors even if they before irrelevant when other regressors are added because of collinearity [3]. So even though forward selection is our only viable subset selection method when dealing with high dimensionality, it might not be effective at picking the best model.

\section{Regression Trees and Random Forest}

As mentioned before, several methods exist for fitting a model of a given data set. We now focus on a method known as Random Forest, which can be used when dealing with high dimensional data.

Amongst the multiple methods in the Tree Class, we only focus on the Random Forest method in the present paper. However, more information on other methods, such as bagging or boosting, can be found in Gareth James' An Introduction to Statistical Learning 
with Applications in $R$ or Kotu's Predictive Analytics and Data Mining. Before we introduce random forests, it is essential to understand the concept of regression trees.

\section{Regression Trees}

Regression Trees are a data mining technique that involves stratifying the predictor space into simpler regions than the original predictor space [9]. We then use the mean of the response variable $y$ in each region as the "fitted" value for any observed data point that falls inside that region. The textbook An Introduction to Statistical Learning with Applications in $R$ provides a simple algorithm for building regression trees without getting into the complexity of the formulas used in it. The regression tree algorithm is provided here:

1. Divide the predictor space into $J$ different non-overlapping regions $R_{1}, R_{2}, \ldots, R_{J}$, known as leaves or terminal nodes.

a. Let $\{X\}$ be the predictor space before any splitting has occurred.

2. For every single observation of the response variable that falls into a region $J$, the "fitted" value of that observation is $\overline{\mathrm{y}}_{R_{j}}$, the mean of all observations in region $R_{j}$, where $1 \leq j \leq J$.

When we think of splitting the predictor space, the questions "which regressor do we split first?" and "at what point should a regressor be split?" arise. In simple terms, we select a predictor variable $X_{j}$ and the cutoff point $c$ such that splitting the predictor space into the regions $\left\{X \mid X_{j}<c\right\}$ and $\left\{X \mid X_{j} \geq c\right\}$ yields the greatest reduction in SSE, the sum of squares

of the error [3]. The stratifying or splitting of the regressor space continues until the SSE can no longer be reduced or until each leaf has no more than five observations [3]. Figure 
5 (left) provides a visual of a regression tree that has been created using two predictor variables while figure 5 (right) is a visual of the generated regions. Note that it looks like an "upside down" tree, hence the name. Although the formulas and algorithm of regression
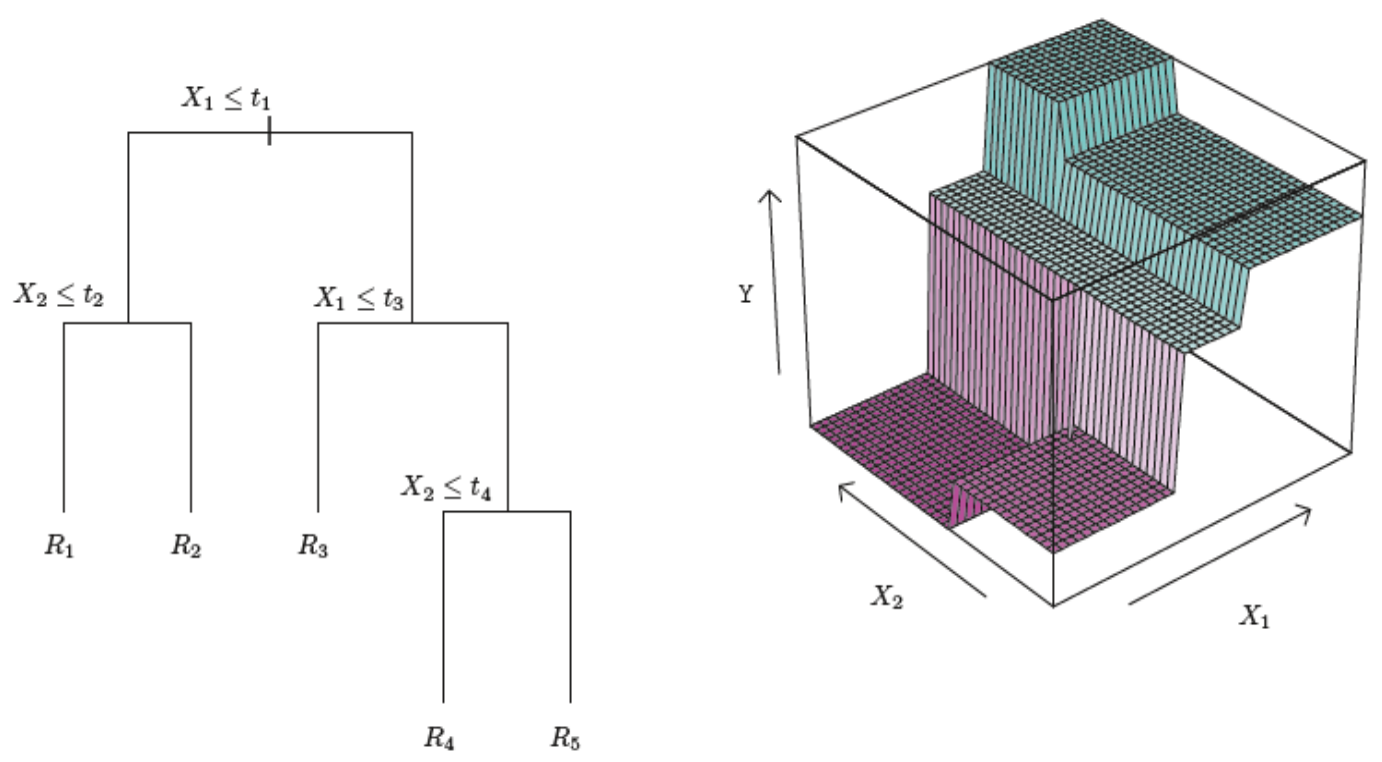

Figure 5: Splitting of a regressor space (left) and the creation of regions in the response variable (right). (Image taken from An Introduction to Statistical Learning with Applications in R)

trees are much more complicated, we only provide a general idea in this paper without loss of generality.

\section{Random Forests}

Recall that regression trees have a predicted value for each region created by the predictor space. In random forests, we obtain multiple de-correlated regression trees and average them [10].

Random Forest is a method that uses multiple trees $T_{1}, T_{2}, \ldots, T_{B}$ to cast an average "fitted" value $f^{B}(x)$ to each observation of the response variable $Y$, where $x$ represents the values 
of a set of predictors that correspond to an observation $y$. The fitted value generated by a random forest for an observation $y_{i}$ is given by:

$$
f_{a v g}^{B}(x)=\frac{1}{B} \sum_{b=1}^{B} T_{b}\left(x, R_{j}\right)=\frac{1}{B} \sum_{b=1}^{B} T_{b j}(x)
$$

where $B$ is the total number of trees and $T_{b}\left(x, R_{j}\right)$ represents the fitted value given by the $j^{\text {th }}$ region in the $b^{\text {th }}$ regression tree for a given set of predictor values $x$ that correspond to observation $y$. In a simpler notation similar to the one provided to regression trees, we can see $T\left(x, R_{j b}\right)$ as $\bar{y}_{R_{j} b}$ for set of predictor values $x$. An advantage of random forests include the fact that they do not overfit the data, no matter how many trees are used [3]. However, it is suggested using $B=100$ to achieve good predicted values of the observed data [3]. Also, random forests use trees that randomly select a subset of predictor variables (usually $1 / 3$ of the original number of regressors), making the trees non-correlated [10]. Even though some regressors may not be optimal to a regression tree, their selection might reveal interaction effects with other predictor variables that would otherwise be ignored if a subset of regressors was not randomly selected [12]. The book The Elements of Statistical Learning (Hastie, Tibshirani, Jerome, 2009) provides a simple algorithm for building a random forest, which is summarized here:

1. For $b=1,2, \ldots, B$, grow a regression tree $T_{b}$ using $l$ randomly selected predictor variables from the total $k$. A typical value for $l$ is $k / 3$.

a. Note: Refer to the previous section on regression trees to know when to stop splitting/stratifying.

2. Ensemble/combine the $B$ trees to create new regions. 
3. Make predictions using the formula $f_{a v g}^{B}(x)=\frac{1}{B} \sum_{b=1}^{B} T_{b}\left(x, R_{j}\right)[10]$.

Suppose we have a response variable $Y$ and three predictor variables $X_{1}, X_{2}, X_{3}$ and $X_{4}$. Suppose also that we decide to make a random forest using two regression trees. The first tree $T_{1}$ is built using randomly selected regressors $X_{1}$ and $X_{3}$, while the second tree $T_{2}$ is built using randomly selected regressors $X_{2}$ and $X_{4}$. Figure 6 offers a visual of this
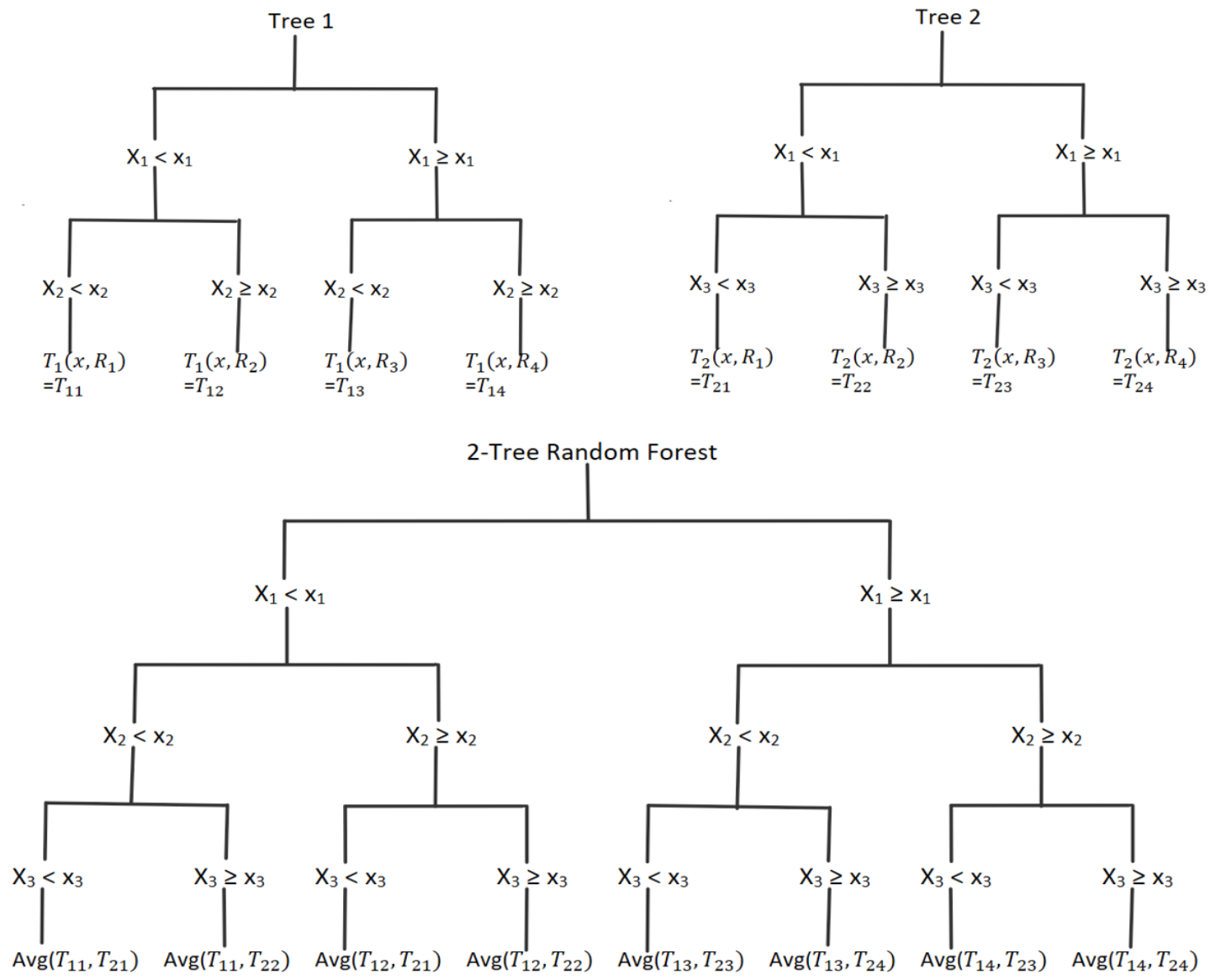

Figure 6: A visual of a Random Forest using two trees. (Note that the fitted value of each region in the forest is the average of two regions: one from each tree. Visual created using the logic presented in this section.)

example using the idea of random forests explained in this section. We can see from the diagram in Figure 6 that the "new regions" have a fitted value equal to the average of the corresponding fitted values in the individual trees. 
It seems that random forests do a good deal of providing fitted values for observed data. However, they do not provide us with a model, but rather a logic for determining the predicted value of $y$ for a given set of predictor values. Here we explore an example of random forests found in Genuer, Poggi, and Tuleau-Malot's "Variable selection using random forests" (2010) found in the Pattern Recognition Letters journal.

\section{Example}

The example stated in the "Variable selection using random forests" article uses the Ozone dataset, located in the mlbench package in statistical program R. The Ozone dataset contains 366 observations, 163 of which contain missing values on at least one variable, so we are left with only 203 observations. The dataset contains a total of 13 variables [13], the fourth of which is used as the response variable and the remaining twelve are the

predictor variables. The example used the suggested $\frac{k}{3}=\frac{12}{3}=4$ predictors per regression tree and grow a total of 2000 trees for the random forest [13], proceeding by creating the random forest and obtaining the regressors that had a positive importance on $Y$-Daily maximum one-hour-average ozone. The important regressors were $X_{1}$-Month, $X_{5}$-Pressure height, $X_{7}$-Humidity, $X_{8}$-Temperature (Sandburg), $X_{9}$-Temperature (El Monte), $X_{11^{-}}$ Pressure gradient, and $X_{12}$-Inversion base temperature [13].

In the next chapter, we provide a new subset selection method that can potentially provide us with a good model, even when we are dealing with high dimensional data. 


\section{CHAPTER II}

\section{NEW SUBSET SELECTION METHOD}

Now that we have a good idea on the concepts of linear regression and some of the many methods that can be used for reducing the dimensions of a data set, we can go over a new method that can potentially provide us with an effective model, whether the data has a low or high dimensionality. We begin with an overview of the new procedure, followed by a detailed explanation of each step along with any comparisons used in each step. The statistical program $\mathrm{R}$ was used to build the procedure. Therefore, we include a quick overview of the program code logic after each step plus a copy of the full program code in Appendix A.

\section{An Overview}

The proposed method consists of selecting various regressors that will make an effective model without having to go through every possible subset like best subset selection; without having to keep previously selected regressors in the model like forward selection; without having to keep previously eliminated regressors out of the model like backward elimination. For naming purposes, we will be using the name Best Probable Subset (BPS) for the name of this new method.

Suppose we want to reduce the dimensions of the regressor space by a certain percent, say $60 \%$. That would mean that we want to keep $40 \%$ of the total number of regressor $k$. Let us say that $40 \%$ of $k$ equals $0.40 * k=l$ regressors that we want to keep in the model. Previously seen methods, like forward selection, backward elimination, best subset selection, and step-wise regression offer ways to select a good subset of regressors $l$. 
However, as previously discussed, they all run into issues, whether computational ineffectiveness, inability to change previously added/deleted regressors, or inability to deal with high dimensional data. The logic behind the BPS method consists of selecting a subset of $l$ regressors using a probability pool that is calculated from the linear relationships - or squared correlations - between the response variable $Y$ and each of the predictor variables. The probability pool, conveniently named Linear Relationship Pool (LRP), is then used to select a subset of $l$ predictors without replacement to avoid selecting the same predictor twice for the same fitted model. The LRP allows us to have models containing regressors that might not be present in other models at all. This solves the issue that we run into in forward selection, in which previously selected predictors have to stay in the fitted model [3].

Once the $l$ regressors are chosen, we use the original LRP for all regressors to have chance of be picked in the next model, even if they were chosen in the previous model, and repeat the selection process until we have 1000 models. Note that each regressor can be in multiple models but the same regressor cannot be more than once in the same model. We decided to use 1000 as the total number of possible models because it gives each predictor a chance of being picked in at least one model, even if they have a squared correlation as small as $\frac{1}{1000}=0.001$ with the response variable. We then calculate the coefficient of determination, $R^{2}$, for each model of $l$ predictor variables and select the model with the highest $R^{2}$ out of the 1000 . The selected model is considered to be the "best probable model" with $l$ regressors using the BPS method, which we denote as $M_{l}^{B P S}$ and the subset of regressors in that model make up the "best probable subset" of size $l$. When dealing with 
high dimensional data, this subset selection procedure is much more computationally efficient than the other subset selection methods mentioned in the previous chapter.

Figure 7 summarizes the entire procedure for finding the best probable fitted model with $l$ regressors. The blue path serves as a guide of the steps of the BPS method while the red path are alternative steps used for comparison purposes. The key next to the diagram provides more information about the colors and shapes. 


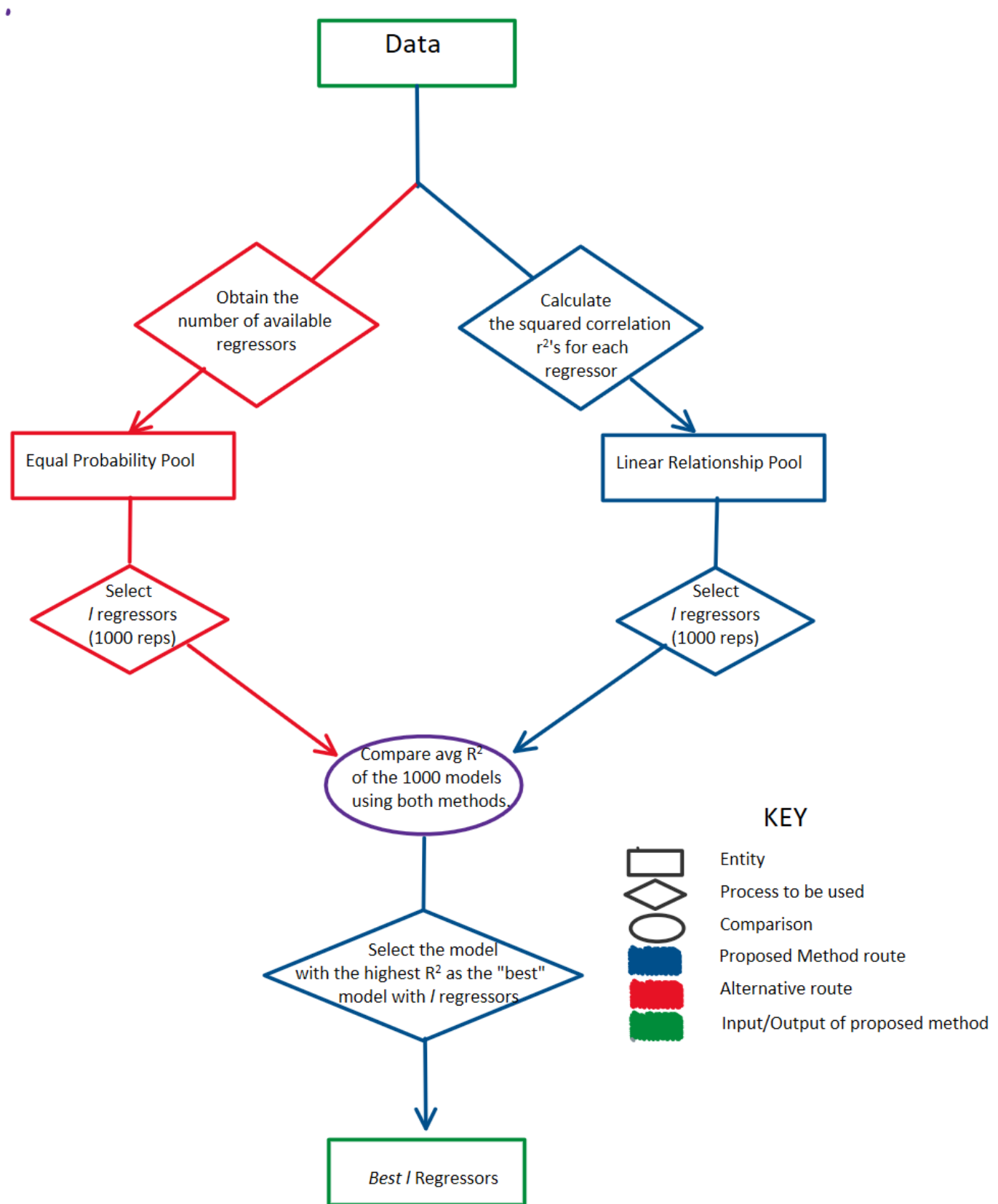

Figure 7: Diagram of the Best Probable Subset (BPS) method. 


\section{Step One: Linear Relationship Pool}

The first step on the process is obtaining our sampling plan, the Linear Relationship Pool (LRP), which is created by calculating the squared correlation, $r^{2}$, of each predictor variable with the response variable. We then use these squared correlations and divide each of them by the sum of all squared correlations, giving us a probability of selection, $p$, for each regressor. Each of these probabilities can be calculated using the formula:

$$
p_{j}=P\left(X_{j}\right)=\frac{r_{j}^{2}}{\sum_{i=1}^{k} r_{i}^{2}}, 1 \leq j \leq k
$$

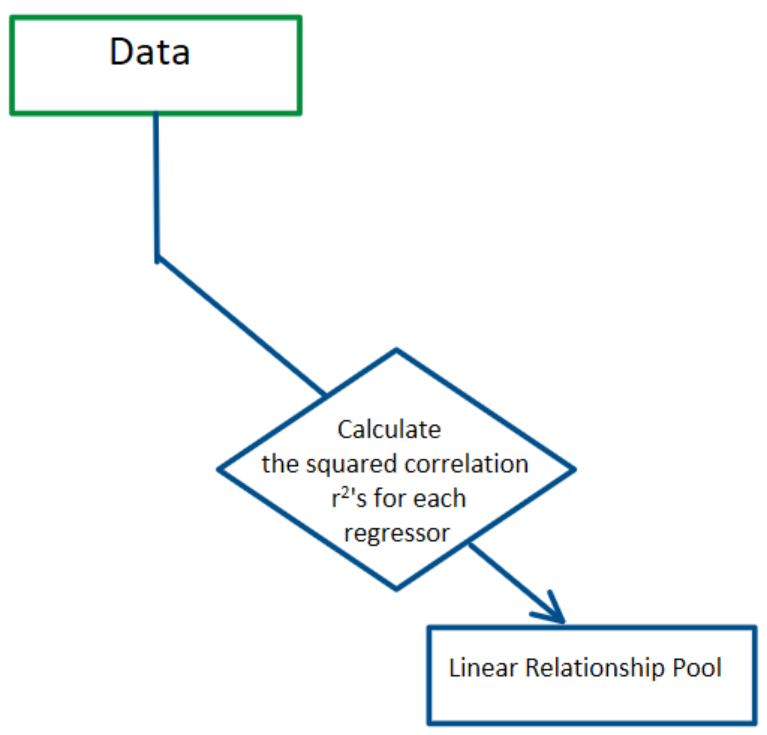

where $k$ is the total number of regressors in the dataset and $r_{j}^{2}$ is the marginal $\mathrm{R}$-squared of the $j^{\text {th }}$ regressor with the response variable $Y$. Once we have calculated every $p_{j} \quad$ for $1 \leq j \leq k$, we have completed the LRP and are ready to proceed to the next step.

Figure 8: Step One: Creating the Linear Relationship Pool (LRP)

\section{Coding Equivalent}

In coding, we create the LRP using the following steps:

1. Calculate the squared correlation of each predictor with the response variable.

2. Calculate the probability of selection, $p$, for each predictor variable. 
3. Find the number of decimal places, $m$, until the first non-zero digit for the smallest probability.

4. Create a vector in which integer $j$ appears $\operatorname{round}\left(p_{j} * 10^{m+1}\right)$ times, representing the $j^{\text {th }}$ regressor. The value $m$ makes sure that the regressor with the smallest probability is represented at least once in the vector.

\section{Example}

Suppose we have three regressors, $X_{1}, X_{2}, X_{3}$ with marginal squared correlations equal to $0.070,0.800$, and 0.002 , respectively. Their probabilities of being selected would be $\frac{0.07}{0.07+0.8+0.002}=0.080,0.917$, and 0.003 , respectively. The smallest probability is 0.003 , which has two decimal places before the first non-zero digit, so $m=2$. In R, we would have a vector where "1" (for Regressor $\left.\quad X_{1}\right) \quad$ appears

$\operatorname{round}\left(0.080 * 10^{2+1}\right)=80$ times, "2" (for Regressor $X_{2}$ ) appears $\quad \operatorname{round}(0.917 *$ $\left.10^{2+1}\right)=917$ times, and "3" (For Regressor $X_{3}$ ) appears $\operatorname{round}\left(0.003 * 10^{1+1}\right)=3$ times. This step completes our Equal Probability Pool
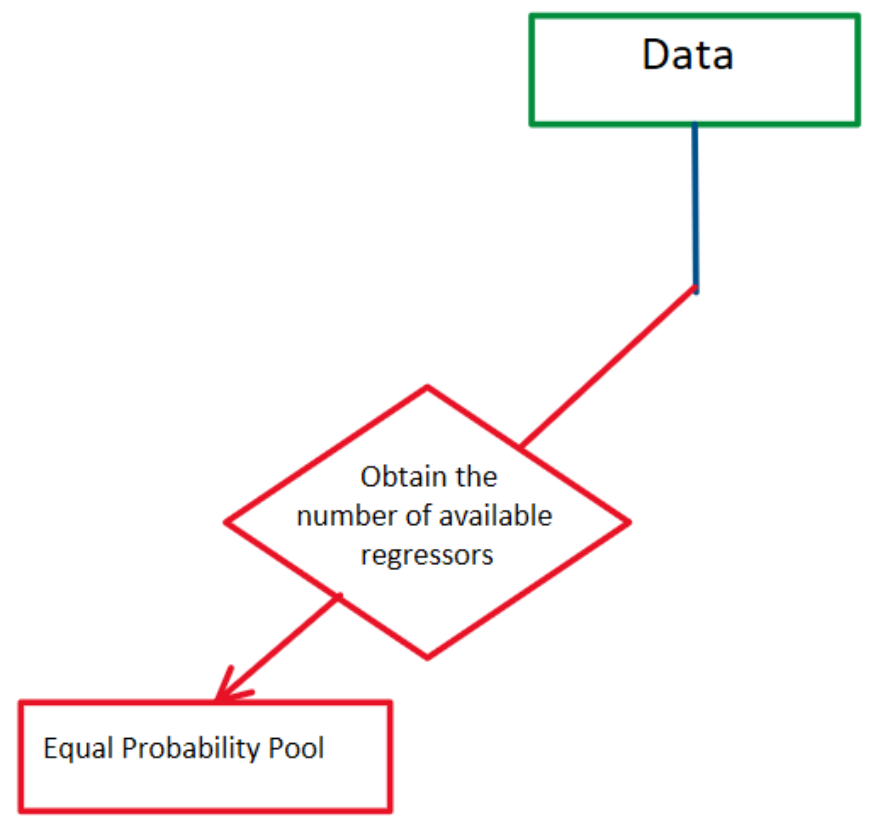

linear relationship pool and the Figure 9: Step One: Alternative sampling plan. vector to be used in $\mathrm{R}$ for the selection of $l$ regressors. 


\section{Comparison Step: Equal Probability Pool}

For comparison purposes, we also use random selection of $l$ regressors without replacement for each of the 1000 fitted models. We use this alternative sampling plan to compare the $R^{2}$ s generated by the 1000 models with $l$ regressors selected using the LRP and the $R^{2}$ s generated by the 1000 models with $l$ regressors selected at random. Equal probabilities means that all predictor variables have the same chance of being selected. Therefore, the probabilities for each regressor is:

$$
p=p_{j}=P\left(X_{j}\right)=\frac{1}{k}, 1 \leq j \leq k
$$

Where $k$ is the total number of regressors and $p_{j}$ is the probability of selecting the $j^{\text {th }}$ regressor.

\section{Coding Equivalent}

In coding, we create the pool of equal probabilities using the following steps:

1. For $j=1,2, \ldots, k$ : create a vector in which integer $j$ appears one time, representing the $j^{\text {th }}$ predictor variable.

a. Note: When coding, we can use $\operatorname{seq}(1: k)$ to create this vector.

\section{Example}

Suppose we have the same three regressors, $X_{1}, X_{2}, X_{3}$ as in the previous example with the same squared correlations $0.070,0.800$, and 0.002 , respectively. Their probabilities of being selected at random would be $1 / 3$ for each of them. Therefore, the vector of integers in $\mathrm{R}$ contains "1", "2", and "3" once each. 


\section{Step Two: Selection of the Predictor Variables}

Now that we have the linear relationship pool, the next step in the procedure is selecting

the $l$ predictor variables using the probabilities. For comparison purposes previously mentioned, we will be selecting a subset of $l$ predictors 1000 times using the LRP and another 1000 times using the equal probabilities pool. Both methods of selection can be done using a computer program. We used computer program $\mathrm{R}$ for both random and nonrandom selections.

\section{Coding Equivalent}

Using Statistical Computer Program R, this can be easily done by using the command:

$$
\text { sample(Prob, } 1, T)
$$

where Prob is the probability pool being used, whether LRP or the equal probabilities pool for selecting at random. This command selects an integer from a vector of integers and deletes all of its repetitions. The "T" - which represents True - deletes any replicates of the selected number. Deleting replicates avoids selecting the same regressor more than once for the same fitted model; it is our way of selecting $l$ regressors without replacement.

\section{Example}

Recall our previous example, where the three regressors, $X_{1}, X_{2}$, and $X_{3}$ have a LRP with probabilities $0.080,0.917$, and 0.003 , respectively, of being selected using the R-Squared Probability pool. Suppose that we want to select $l=2$ out of the three total regressors. Let us assume that $X_{2}$ is selected first since it has a significantly larger probability of being than the others. Using sample $(L R P, 1, T)$, once the integer "2" is selected - which 
represents regressor $X_{2}$ - the 917 repetitions of the integer "2" will be deleted from the vector of integers, leaving us with 80 repetitions of integer "1" and 3 repetitions of integer "3". Since we are selecting two regressors without replacement, every time we select another regressor for the fitted model the probabilities of those regressors that have not yet been selected are updated. This example is just for the sake of showing the logic of the method. It would be totally unnecessary to produce 1000 models with $l=2$ out of three regressors.

Comparing the Outcome of Using both Probability Pools

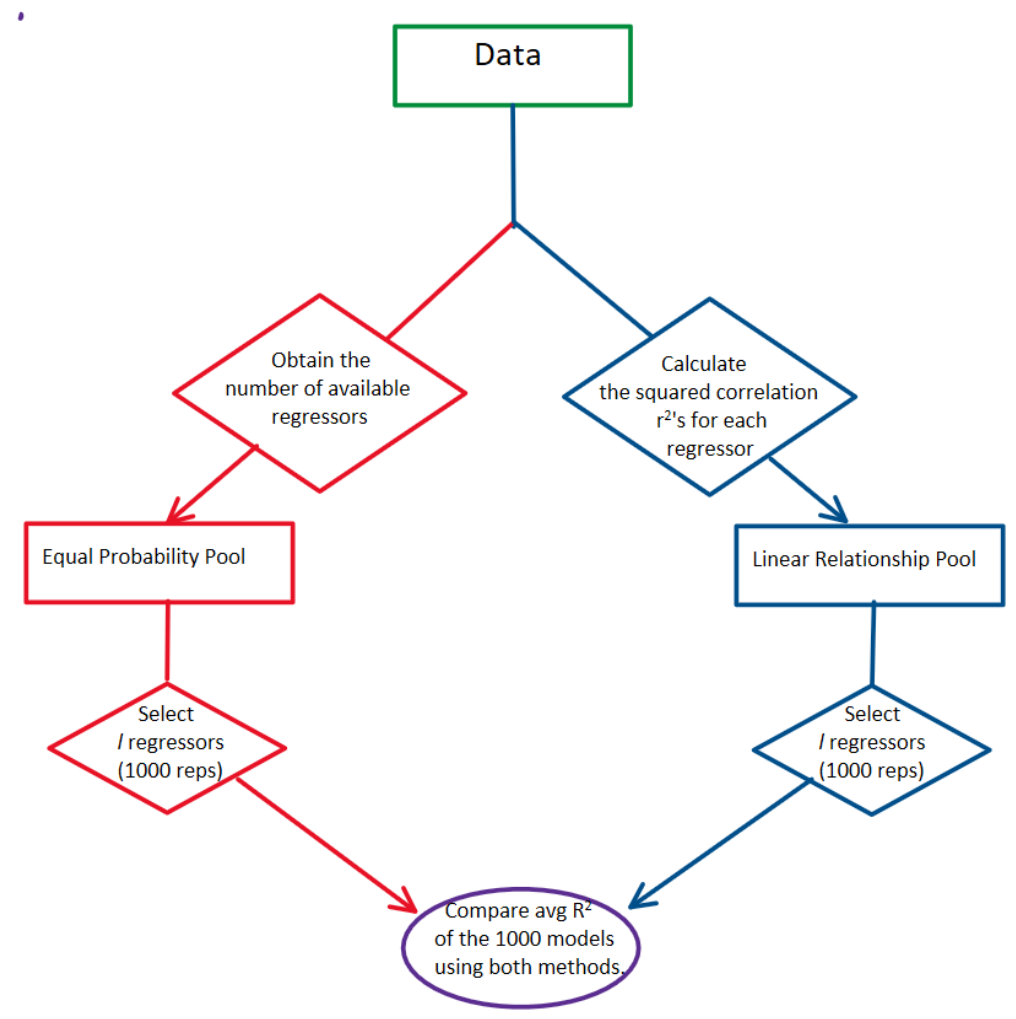

Figure 10: Step Two: Selecting a subset of l regressors using the preferred and alternative sampling plans and a comparison between the two.
We have been using two probability pools -the LRP and the equal probabilities pool- to select $l$ out of $k$ regressors. In order to determine whether which probability pool is indeed more effective, we only need to compare the average R-Squared of the 1000 fitted models

produced by selecting $l$ regressors using the LRP and the average R-Squared of the 1000 
fitted models produced by selecting a subset of $l$ regressors using the random probability pool. In short, we just need to show that:

$$
\operatorname{Avg}\left(R_{L R P}^{2}\right)>\operatorname{Avg}\left(\left(R_{E P P}^{2}\right)\right.
$$

Where LRP is means "linear relationship pool" and EPP means "equal probabilities pool" for selecting at random. We should expect to obtain models with higher $R^{2} \mathrm{~s}$ when using the LRP since the regressors that have a higher squared correlation with the response variable have a higher chance of being selected in the subset of $l$ regressors. We omit showing this in the examples in Chapter IV, but can be demonstrated using with the code provided in Appendix A.

It should be clear that using the LRP is more effective at creating 1000 fitted models with $l$ regressors having a higher average $R^{2}$ than the 1000 fitted models with $l$ regressors chosen at random. We now continue to the next step in the BPS method assuming that only the LRP will be used for the selection of $l$ regressors. 


\section{Step Three: Choosing the Model with the Best l Regressors}

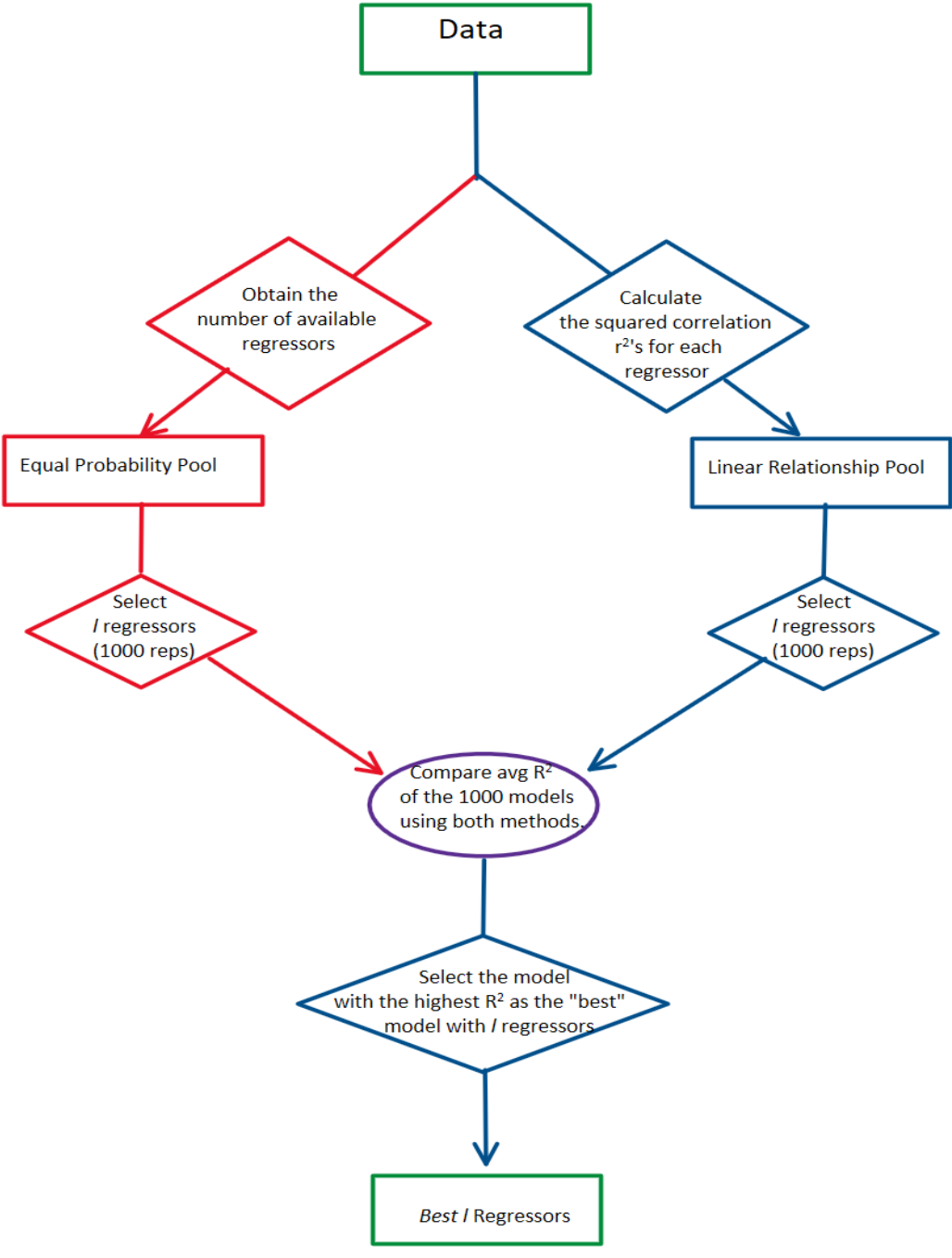

Figure 11: Step Three: Selecting the model with the best subset of $l$.
After creating the
linear relationship
pool and using it to
select a subset of $l$
regressors when
fitting the 1000
models, the next
step is to determine
which of the 1000
models has the best
subset of $l$ out of
the $k$
regressors.
choose the best
model, we must

first decide what

criteria would make one model to be the best out of the 1000 .

\section{Criterion for Selecting the Best Subset of Size l}

We covered in Chapter I that some measures of fit include Mean Squared Error (MSE), the coefficient of determination $R^{2}$, and the $R_{a d j}^{2}$. We also mentioned that $R^{2}$ and adjusted $R^{2}$ have the advantage of having a range between 0 and 1 and less than 1 , respectively, 
making them easier to understand that MSE [3]. Lastly, we explained that $R^{2}$ continues to increase when we keep adding more regressors to the model, making it more useful for comparing fitted models with the same number of regressors.

The dilemma we currently have is determining which model(s) out of the generated 1000 is the best probable model, $M_{l}^{B P S}$, containing the best subset of $l$ predictors. Since all of them have the same number of predictors $l$, we can use the regular $R^{2}$ to measure their fit. Since a large $R^{2}$ indicates a better fit for equally sized models, we just need to select the model with $l$ regressors that yields the highest $R^{2}$.

\section{Coding Equivalent}

Now that we have chosen a measure of fit as the criteria for choosing the best fitted model with $l$ predictor variables, we only need to find out which model has the highest $R^{2}$. In order to do this using $\mathrm{R}$, we simply calculated the $R^{2}$ for each of the 1000 models having $l$ predictors and sorted them from largest to smallest using the command:

$$
\operatorname{sort}(R S q u a r e s, \text { decreasing }=T R U E)
$$

where RSquares is a vector containing the $1000 R^{2}$ and the subsection "decreasing = TRUE" tells R to sort the numbers from largest to smallest. We select the highest $R^{2}$ and the unique model that yields it.

\section{Step Four: Determining the Best Subset Size l}

Up to now we have assumed that the reduction amount that we want to perform on a data is known, making it clear what the subset size $l$ is. But what happens if we do not know what the reduction amount is? Or what if we want to select the best subset, no matter what the size of it is? Assuming that the sample size $n$ is much greater than the number of 
regressors $k$, we are left with $k+1$ possible best probable models $-M_{0}^{B P S}, M_{1}^{B P S}, \ldots, M_{k}^{B P S}$ each of them being the best probable subset of size $0,1, \ldots, k$, respectively - and no direction on where to go next. We proceed by determining a criteria for selecting the single best probable subset without having a pre-set subset size.

\section{General Criterion for Selecting the Best Subset of Any Size}

In the previous section, we used the R-Squares to determine the best fitted linear model out of the 1000 models generated using the Linear Relationship Pool. Assuming that we fit a model with the best probable subset for all possible subset sizes, we are left with $M_{0}^{B P S}, M_{1}^{B P S}, \ldots, M_{k}^{B P S}$, a total of $k+1$ plausible models, where $M_{0}^{B P S}$ is the null model and $M_{k}^{B P S}$ is the full model containing all regressors. Since the $k+1$ best probable models contain different number of regressors, we need other criteria other than the $R^{2} \mathrm{~s}$ to determine the best one out of them.

As mentioned in Chapter I, the $R^{2}{ }_{a d j}$ statistic offers flexibility when comparing subsets of different sizes because, unlike the non-adjusted $R^{2}$, it is penalized for the addition of unnecessary regressors [3]. Furthermore, $R^{2}$ adj is simple to understand as it goes up to 1. Because of these reasons, we decided to use this statistic as the criterion for choosing the best possible subset out of the $k+1$ subsets to consider.

The $R^{2}$ adj increases when an added regressor contributes to the fit of the model, so the best out of the $k+1$ plausible fitted models $M_{0}^{B P S}, M_{1}^{B P S}, \ldots, M_{k}^{B P S}$ should be the one yielding the highest $R^{2}{ }_{a d j}$. We will use the fitted model having the highest $R^{2}{ }_{a d j}$ as the general criteria for choosing the best probable subset. We denote the linear model containing the best probable subset as $M^{B P S}$, without a subscript. 


\section{Coding Equivalent}

Similarly to the coding logic in the previous step, we simply calculate the $R^{2}$ adj for each of the $k+1$ models, each having the best probable subset of size $0,1, \ldots, k$ and sort the adjusted $R^{2}$ s using the command:

$$
\operatorname{sort}(\text { AdjustedRSquares, decreasing }=T R U E)
$$

where AdjustedRSquares is a vector containing the $k+1 R^{2}{ }_{a d j}$ and the subsection "decreasing = TRUE" tells R to sort the numbers from largest to smallest. We select the highest adjusted $R^{2}$ and the unique model that yields it, $M^{B P S}$.

\section{High Dimensional Data and Stopping Rules}

Recall from Chapter I that data having a large number of predictors, $k$, that equals or exceeds the number of observations, $n$, are known to have high dimensionality, and that the least squares estimation does not work with such data [3]. Recall also that forward selection is the only alternative subset selection methods when we are dealing with such data. Here we explain how the best probable subset method deals with high dimensional data while keeping the number of fitted models to a minimum by creating some stopping rules.

\section{Stopping Rules}

We begin by creating a stopping rule so that the number of predictors in the model never exceeds, or even approaches, the number of observations. For this paper, we want the number of observations to be at least 5 times larger than the number of regressors in the fitted mode, limiting $l$ to be less than or equal to $n / 5$. The rule will allow us to have five observations per regressor and let us always use the BPS method while dealing with low 
or high dimensional data. Note that the $n / 5$ limit on $l$ can be changed to any other number less than $k$, and there is no statistical proof that this limit will always be the best; the limit is specified for the purpose of this paper can be changed accordingly as long as it is less than the number of observation $n$.

Using the stopping rule above, we would still need to consider various best probable models of different sizes, $M_{0}^{B P S}, M_{1}^{B P S}, \ldots, M_{n / 5}^{B P S}$. If $n / 5$ were to equal 100 , we would still be considering $100+1$ best possible models of different sizes, each of which was selected from 1000 models, with the exception of the null model $M_{0}^{B P S}$. The total number of fitted models would then equal $100 * 1000+1=100,001$, which can be computationally inefficient. To improve the situation, we created a second stopping rule, which states that we will continue to find the best possible subset of the next size until the adjusted R-squared ceases to increase by more than $1 \%$. In short, we begin by finding $M_{0}^{B P S}$, followed by $M_{1}^{B P S}$, followed by $M_{2}^{B P S}$, etc. until $M_{i}^{B P S}$ yields a lower $R^{2}{ }_{a d j}$ than $M_{i-1}^{B P S}$, where $1 \leq i \leq$ $k$. At that point $M_{i-1}^{B P S}$ is the best probable model. Note that the $1 \%$ was selected for the present paper but there could be other stopping rules that are just as reliable.

When we combine the stopping rules described above, we obtain the following stopping criteria:

1. Begin by finding the null model $M_{0}^{B P S}$.

2. For $1 \leq i \leq \min \left(k, \frac{n}{5}\right)$, continue finding the model with the best possible subset size $i$ until $M_{i}^{B P S}$ yields an $R^{2}{ }_{a d j}$ than is less than $1 \%$ greater than the $R^{2}{ }_{a d j}$ of $M_{i-1}^{B P S}$ 
Once we have reached the stopping rule, we are left with $i+1$ best possible models of different sizes. Since the adjusted R-squared continues to increase by more than $1 \%$ until $M_{i-1}^{B P S}$, the best probable model is $M_{i-1}^{B P S}$, so $M^{B P S}=M_{i-1}^{B P S}$.

\section{Best Probable Method Algorithm}

The Best Probable Subset (BPS) method is summarized here:

1. Create a probability pool, called Linear Relationship Pool (LRP), for selecting a subset of regressors by using the following formula for each regressor of the $k$ regressors.

$$
p_{j}=P\left(X_{j}\right)=\frac{r_{j}^{2}}{\sum_{i=1}^{k} r_{i}^{2}}, 1 \leq j \leq k
$$

Where $p_{j}$ is the probability of choosing the $j^{\text {th }}$ predictor for the reduced model and $r_{j}^{2}$ represents the squared correlation between the $j^{\text {th }}$ predictor and the response variable.

2. Begin by finding the null model $M_{0}^{B P S}$. For $1 \leq i \leq \min \left(k, \frac{n}{5}\right)$ :

a. Select a subset of size $i$ without replacement from the predictor by using the LRP. Repeat 1000 times and select the model with the highest $R^{2}$. Call it $M_{i}^{B P S}$

b. Continue finding the model with the best probable subset size $i$ until $M_{i+1}^{B P S}$ yields an $R^{2}{ }_{a d j}$ than is less than $1 \%$ greater than the $R^{2}{ }_{a d j}$ of $M_{i}^{B P S}$.

3. Since the adjusted R-squared continues to increase by more than $1 \%$ until $M_{i-1}^{B P S}$, the best probable model is $M^{B P S}=M_{i}^{B P S}$. 


\section{CHAPTER III}

\section{DATA SIMULATION}

In order to validate the BPS method, we simulated some linear regression models and generated data from them. In Chapter III we cover how we generated both the models and the data.

\section{Simulating Multiple Regression Linear Models}

Our next goal is to prove that the BPS method can pick up a subset of regressors that make an effective fitted model for observed data. To do so, we create multiple regression linear models and generate "observed" data from them. Without loss of generality, we decided to create simulate three linear models, each containing a different total number of regressors - $k=10,25$, and 50. To create data sets containing significant and insignificant regressors, we made the response variable using only the first $20 \%$ of the regressors. The data for each regressor, whether significant or insignificant to the model, were generated using one randomly chosen distribution from this list:

Chi-Squared (DF is a random integer from 1 to 10$)$

$>$ Gamma ( $\alpha$ is a random integer from 1 to $10 ; \beta$ is a random integer from 1 to 10 ) Normal ( $\mu$ is a random integer from 1 to $10 ; \sigma$ is a random integer from 1 to 10 )

Once the data for the first $20 \%$ of the regressors are generated, the observations for the response variable were created using the model:

$$
Y=\beta_{1} X_{1}+\beta_{2} X_{2}+\cdots+\beta_{l} X_{l}+\varepsilon
$$


where $X_{1}, X_{2}, \ldots, X_{l}$ are the first $20 \%$ and only significant regressors and the regression parameters are randomly generated integers from -10 to 10 , excluding 0 . The error term $\varepsilon$ is distributed as $N\left(0, \sigma^{2}\right)$, where $\sigma^{2}$ is specified in each dataset. Once the observations of the response variable are generated using the above model, we simulate data for the other $80 \%$ of the regressors, each having a regression parameter equal to zero and a distribution chosen at random from the list:

$>$ Uniform (where $\mathrm{x}$ is between 0 and 1 )

Poisson ( $\lambda$ is a random integer from 1 to 10$)$

Binomial ( $\mathrm{p}$ is a random number between 0 and $1 ; \mathrm{n}$ is a random integer form 1 to 10)

Beta ( $\alpha$ is a random integer from 1 to $100 ; \beta$ is a random integer from 1 to 10 )

$>$ Chi-Squared (DF is a random integer from 1 to 10$)$

Exponential ( $\beta$ is a random integer from 1 to 10$)$

$>$ Gamma ( $\alpha$ is a random integer from 1 to $100 ; \beta$ is a random integer from 1 to 10 )

Normal ( $\mu$ is a random integer from 1 to $100 ; \sigma$ is a random integer from 1 to 10 )

To compare the BPS method with already existing methods, we need the number of observations to be well above the number of regressors. The three data sets mentioned in this section will have a sample size equal to five times the total number of regressors, $k$.

\section{A Data Set with High Dimensionality}

We created a $4^{\text {th }}$ data set, also having a total of 100 regressors, which have 80 observations and only the first 20 regressors will be significant to the model. We will use this high 
dimensional data set to compare it to forward selection and random forests, both of which can be used in data with high dimensionality.

Lastly, we reference back to the random forest example provided at the end of Chapter I, and it to the BPS method by looking at the MSE that each of the two methods produced using the Ozone dataset.

\section{DATA COLLECTION}

The section walks the reader through some existing data sets used in this paper. The data set, named "U.S. News and World Report's College Data," was collected from the "ISLR" package in $\mathrm{R}$, and belong to their respective publishers. The data set, along with many others, was made public and can be used freely. Without further delay, here is a summary of the data:

\section{“College" - U.S. News and World Report's College Data}

The College data set offers statistics for a large number of Colleges, including graduation rates, enrollments, tuition, and more. The data was collected from the 1995 US News and World Reports[11]. The following information is a summary from the dataset description in R:

R Package

"ISLR"

Description

The College data has 777 observations and 18 variables.

Format

This data frame contains the following columns: 
“Private Indicates 'Yes' or 'No' as a factor

Apps Number of applications received

Accept Number of applications accepted

Enroll Number of new students enrolled

Top10perc Percent of new students from the top $10 \%$ of their H.S class

Top25perc Percent of new students from the top $25 \%$ of their H.S class

F.Undergrad Number of full time undergraduates

P.Undergrad Number of part time undergraduates

Outstate Out-of-state tuition

Room.Board Costs for room and board

Books Estimated cost of books

Personal Estimated amount of personal spending

PhD Percent of faculty with Ph.D.'s

Terminal Percent of faculty with terminal degrees

S.F.Ratio Student/faculty ratio

Perc.alumni Percent of alumni who donate to the College

Expend Instructional expenditure per student

Grad.Rate Graduation rate" [11].

Source

Dataset was taken from the Statistics library, StatLib, maintained at Carnegie Mellon University. The dataset was also used in the 1995 Data 
Analysis Exposition, sponsored by the ASA Statistical Graphics Section

[11].

For more information on this dataset, refer to the ISLR package documentation. 


\section{CHAPTER IV}

\section{COMPARISONS AND RESULTS}

In Chapter IV we apply the BPS and other existing methods to each data set to provide some insight on the method's functionality. In the first section, we use simulated data sets that follow the linear regression assumptions in order to validate the new method and contain low to moderate correlation between regressors. After, we use a data set that is not as perfect and compare the results with some existing methods.

\section{Validation of the BPS Method}

We begin the validation of the BPS method by using a simulated data sets with only ten predictor variables. For each data set we use, we provide a list of the contributing regressors, which are those that were used to build the observations of the response variable $Y$. Since we are simulating the data using linear regression models with the error term distributed as $N\left(0, \sigma^{2}=1\right)$, there is no need to check the model assumptions. We do provide the correlation matrix to the first data set. After that, the correlation matrices are too large, so we only provide the highest correlation coefficient between two different regressors to show that there is no collinearity amongst regressors. 


\section{Dataset \# 1:}

Number of Predictors: 10

Contributing Predictors: $X_{1}, X_{2}$

Sample Size: 50

Error Term: $\varepsilon \sim N\left(0, \sigma^{2}=2\right)$

\section{Correlation Matrix:}

\begin{tabular}{|c|c|c|c|c|c|c|c|c|c|c|}
\hline & $x 1$ & $\mathrm{X} 2$ & $\mathbf{x} 3$ & $\mathrm{X} 4$ & $\mathbf{x} 5$ & x6 & $\mathbf{x} 7$ & $\mathrm{x} 8$ & x9 & $\mathbf{x} 10$ \\
\hline $\mathbf{X} 1$ & 1.000 & -0.037 & 0.129 & 0.085 & 0.316 & 0.031 & 0.165 & 0.170 & -0.001 & 0.022 \\
\hline $\mathrm{X} 2$ & -0.037 & 1.000 & 0.043 & 0.042 & 0.294 & -0.029 & 0.177 & -0.080 & -0.027 & -0.135 \\
\hline $\mathrm{X} 3$ & 0.129 & 0.043 & 1.000 & 0.265 & -0.051 & 0.017 & 0.082 & 0.230 & -0.034 & 0.048 \\
\hline $\mathrm{X} 4$ & 0.085 & 0.042 & 0.265 & 1.000 & 0.115 & 0.095 & -0.079 & 0.135 & -0.197 & 0.166 \\
\hline$\times 5$ & 0.316 & 0.294 & -0.051 & 0.115 & 1.000 & -0.248 & 0.147 & 0.121 & 0.031 & -0.148 \\
\hline$x 6$ & 0.031 & -0.029 & 0.017 & 0.095 & -0.248 & 1.000 & -0.042 & -0.094 & 0.118 & 0.156 \\
\hline$x 7$ & 0.165 & 0.177 & 0.082 & -0.079 & 0.147 & -0.042 & 1.000 & -0.080 & 0.024 & -0.167 \\
\hline$x 8$ & 0.170 & -0.080 & 0.230 & 0.135 & 0.121 & -0.094 & -0.080 & 1.000 & -0.087 & -0.075 \\
\hline X9 & -0.001 & -0.027 & -0.034 & -0.197 & 0.031 & 0.118 & 0.024 & -0.087 & 1.000 & -0.111 \\
\hline $\mathrm{X} 10$ & 0.022 & -0.135 & 0.048 & 0.166 & -0.148 & 0.156 & -0.167 & -0.075 & -0.111 & 1.000 \\
\hline
\end{tabular}

Table 1: Correlation Matrix - Dataset \# 1

\section{Non-Zero Correlation Coefficients:}

The following table provides the distribution and true regression coefficients for the regressors with non-zero regression coefficients in Dataset \# 1. All other regressors have a true regression coefficient of zero.

\begin{tabular}{|l|l|r|} 
Regressors & Distribution & Coefficients \\
\hline$X 1$ & X1 NORMAL $(M u=7$, Sigma =9) & 8 \\
\hline$X 2$ & X2 CHI-SQUARED $(d f=10)$ & 5 \\
\hline
\end{tabular}

Table 2: Regression Coefficients - Dataset \# 1 


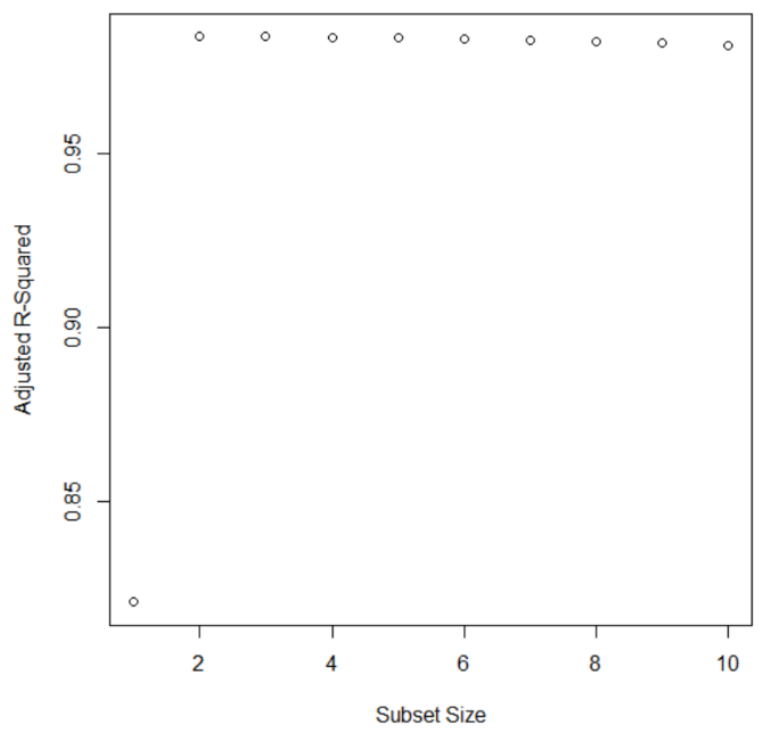

Figure 12: Adjusted R-Squares vs. Subset Size Plot-Dataset \# 1

Using the statistical program $\mathrm{R}$, we generated 1000 models for each subset size $i$, where $1 \leq i \leq 10$. From each group of 1000 models, the best model of size $i$, denoted as $M_{i}^{B P S}$, is selected. The following plot and table show the adjusted R-squares for each $M_{i}^{B P S}$ model.

\begin{tabular}{|c|c|c|c|c|c|}
\hline & SubsetSize & Regressors & RSquares & AdjustedRSquares & AdjRSquaresPercentChange \\
\hline 1 & 1 & 1 & 0.8249 & 0.8212 & NA \\
\hline 2 & 2 & 2,1 & 0.9843 & 0.9836 & 19.8 \\
\hline 3 & 3 & $3,1,2$ & 0.9846 & 0.9836 & 0.0 \\
\hline 4 & 4 & $1,2,8,3$ & 0.9848 & 0.9834 & 0.0 \\
\hline 5 & 5 & $3,8,1,4,2$ & 0.9849 & 0.9832 & 0.0 \\
\hline 6 & 6 & $1,8,4,2,3,7$ & 0.9849 & 0.9828 & 0.0 \\
\hline 7 & 7 & $1,2,8,4,3,7,10$ & 0.9850 & 0.9825 & 0.0 \\
\hline 8 & 8 & $2,1,5,7,4,3,10,8$ & 0.9850 & 0.9821 & 0.0 \\
\hline 9 & 9 & $2,1,7,5,4,8,3,10,6$ & 0.9850 & 0.9817 & 0.0 \\
\hline 10 & 10 & $2,1,5,7,4,3,10,8,9,6$ & 0.9850 & 0.9812 & 0.0 \\
\hline
\end{tabular}

Table 3: Best Probable Subset of Size l-Dataset \# 1

Note that the AdjRSquaresPercentChange contains the percent changes of the $R_{a d j}^{2}$. According to our stopping rule, we should select the best probable subset of two regressors, $X_{1}$ and $X_{2}$, which yield the following LS estimates: 


\begin{tabular}{|r|l|l|l|l|} 
& Estimate & Std. Error & \multicolumn{1}{|l}{ t value } & $\operatorname{Pr}(>|\mathrm{t}|)$ \\
\hline (Intercept) & 0.2532860 & 0.49237050 & 0.5144216 & $6.093678 \mathrm{e}-01$ \\
\hline X1 & 0.9847962 & 0.01954545 & 50.3849292 & $1.466718 \mathrm{e}-42$ \\
\hline X2 & 0.9903822 & 0.04541472 & 21.8075137 & $3.170479 \mathrm{e}-26$
\end{tabular}

Table 4: Best Probable Subset LS Estimates - Dataset \# 1

When we compare the fitted model to the population linear regression model, we can see that we obtained the same regressors, but very different estimates for the linear regression coefficients. The following $R_{a d j}^{2}$ table contains useful information for comparing the different subset selection methods. The table provides the $R_{a d j}^{2}$ for each subset size using each subset selection method. The cells highlighted in yellow indicates the $R_{a d j}^{2}$ value for the best model selected by each method.

\begin{tabular}{r|r|r|r|r|r|r}
\hline & SubsetSize & BPS & BestSubset & Forward & Backward & Stepwise \\
\hline 1 & 1 & 0.8212 & 0.8212 & 0.8212 & 0.8212 & 0.8212 \\
\hline 2 & 2 & 0.9836 & 0.9836 & 0.9836 & 0.9836 & 0.9836 \\
\hline 3 & 3 & 0.9836 & 0.9836 & 0.9836 & 0.9836 & 0.9836 \\
\hline 4 & 4 & 0.9834 & 0.9834 & 0.9834 & 0.9834 & 0.9834 \\
\hline 5 & 5 & 0.9832 & 0.9832 & 0.9832 & 0.9832 & 0.9830 \\
\hline 6 & 6 & 0.9828 & 0.9828 & 0.9828 & 0.9828 & 0.9828 \\
\hline 7 & 7 & 0.9825 & 0.9825 & 0.9825 & 0.9825 & 0.9825 \\
\hline 8 & 8 & 0.9821 & 0.9821 & 0.9821 & 0.9821 & 0.9821 \\
\hline 10 & 9 & 0.9817 & 0.9817 & 0.9817 & 0.9817 & 0.9817 \\
\hline 10 & 0.9812 & 0.9812 & 0.9812 & 0.9812 & 0.9812 \\
\hline
\end{tabular}

Table 5: Subset Selection Methods $R_{a d j}^{2}$ Comparisons - Dataset \# 1

At first glance, we can observe that the BPS method has the same $R_{a d j}^{2}$ values as most subset selection methods for all subset sizes. When we look at the table, we can see that the largest $R_{a d j}^{2}$ for all methods occurs when the subset size equals 2 . According to the subset selection criteria explained in chapter 1, all pre-existing subset selection methods should choose $l=2$ as the best subset size. The BPS method does not take any more 
regressors once the adjusted R-Squared value increases by less than $1 \%$. In this case, the $R_{\text {adj }}^{2}$ increased $\frac{0.9846-0.9843}{0.9843} * 100 \%=0.03 \%$, so we stay at $l=2$ regressors in the best probable model.

Lastly, we compare the Mean Square Error (MSE) of the best probable model with the MSE that we get by building a random forest, which we denote as $M S E_{B P S}$ and $M S E_{R F}$, respectively. Recall from chapter 1 that the suggested number of trees for random forests is 100 and that the number of regressors that should be considered at each node is approximately $n / 3$ for regression random forests. We provide a summary table of the random forest we obtained using dataset \# 1:

The $M S E_{B P S}$ we obtained for dataset \# 1 equals 1.2208 , a much lower number than

\begin{tabular}{|l|r|}
\hline Measure & Value \\
\hline Number of Trees & 100.00000 \\
\hline Splits per Node & 3.00000 \\
\hline MSE & 34.84177 \\
\hline
\end{tabular}

Table 6: Random Forest Summary - Dataset \# 1

$M S E_{R F}=34.8418$. Note that the BPS method is more effective than Random Forests in this data set. This does not mean or imply, however, that the BPS will always be more effective at predicting the values of the response variable. 


\section{Dataset \# 2:}

Number of Predictors: 25

Contributing Predictors: $X_{1}, X_{2}, \ldots, X_{5}$

Sample Size: 125

Error Term: $\varepsilon \sim N\left(0, \sigma^{2}=5\right)$

Maximum Pair-Wise Correlation Value: 0.273

Non-Zero Regression Coefficients:

\begin{tabular}{|r|l|l|r|} 
& Regressors & Distribution & Coefficients \\
\hline 1 & X1 & X1 NORMAL $(M u=3$, Sigma =6) & 3 \\
\hline 2 & X2 & X2 GAMMA(alpha $=7$, beta $=8)$ & 10 \\
\hline 3 & X3 & X3 CHI-SQUARED $(d f=4)$ & 8 \\
\hline 4 & $X 4$ & X4 NORMAL $(M u=3$, Sigma $=2)$ & 6 \\
\hline 5 & X5 & X5 CHI-SQUARED $(d f=1)$ & 4 \\
\hline
\end{tabular}

Table 7: Regression Coefficients - Dataset \# 2

Similar to the first data set, we generated 1000 models for each subset size $i$, where $1 \leq$ $i \leq 25$, using R. From each group of 1000 models, the best model of size $i$, denoted as $M_{i}^{B P S}$, is selected. The following plot and table show the adjusted R-squares for each $M_{i}^{B P S}$.

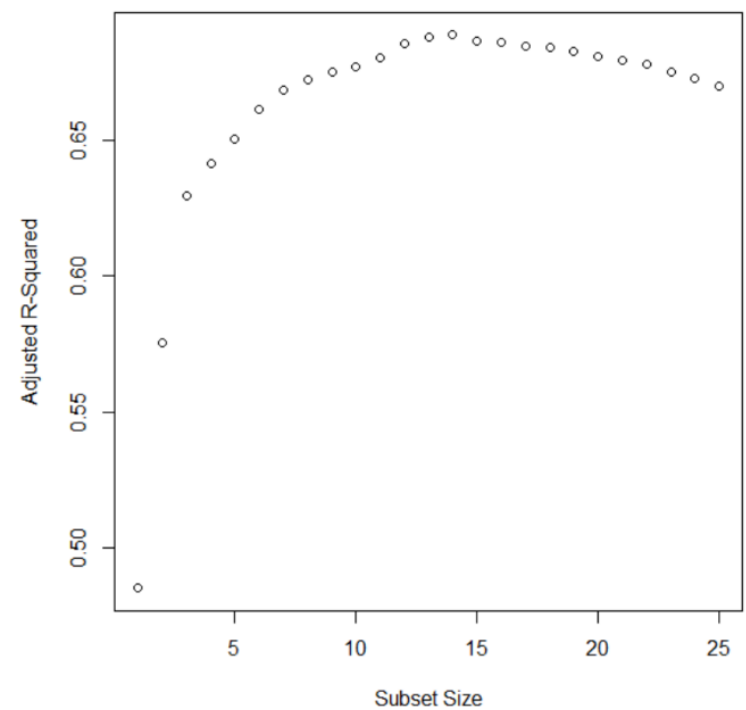

Figure 13: Adjusted R-Squares vs. Subset Size Plot-Dataset \# 2 


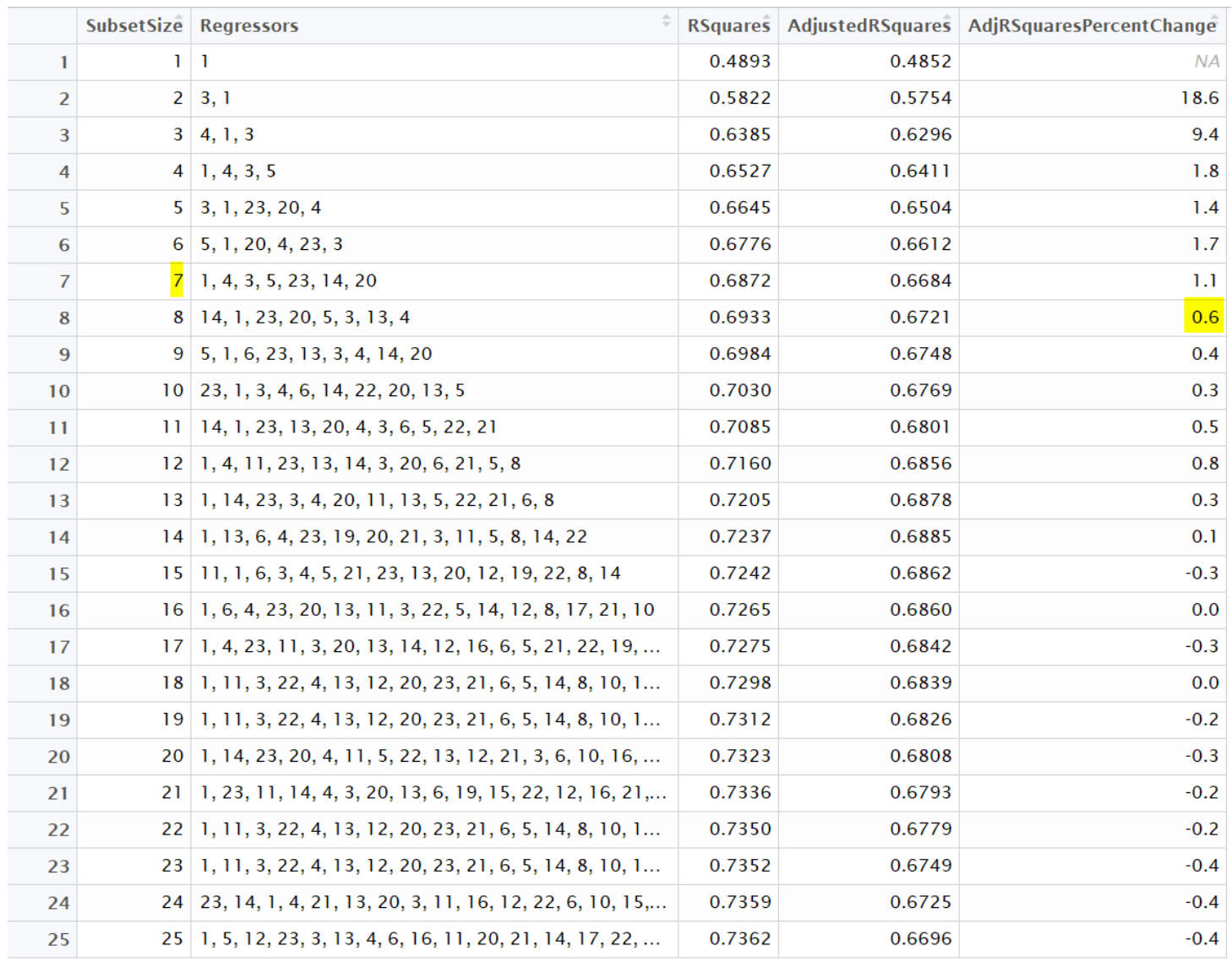

Table 8: Best Probable Subset of Size l-Dataset \# 2

According to our stopping rule, we should select the best probable subset of seven

regressors, $X_{1}, X_{3}, X_{4}, X_{5}, X_{14}, X_{20}$, and $X_{23}$, which yield the following LS estimates:

\begin{tabular}{|r|r|l|l|l|}
\hline & Estimate & Std. Error & t value & $\operatorname{Pr}(>|t|)$ \\
\hline (Intercept) & 5.4416323 & 2.15199293 & 2.528648 & $1.278205 \mathrm{e}-02$ \\
\hline $\mathbf{X 1}$ & 0.9673448 & 0.07380813 & 13.106211 & $1.055851 \mathrm{e}-24$ \\
\hline $\mathbf{X} 3$ & 0.8249247 & 0.15185295 & 5.432392 & $3.068362 \mathrm{e}-07$ \\
\hline $\mathbf{X} 4$ & 1.0004758 & 0.24462950 & 4.089760 & $7.960643 \mathrm{e}-05$ \\
\hline $\mathbf{X} 5$ & 0.8227310 & 0.36204690 & 2.272443 & $2.488585 \mathrm{e}-02$ \\
\hline $\mathbf{X} 14$ & -2.2941722 & 1.21251759 & -1.892073 & $6.095289 \mathrm{e}-02$ \\
\hline $\mathbf{X} 20$ & 0.1779347 & 0.06918388 & 2.571910 & $1.136595 \mathrm{e}-02$ \\
\hline $\mathbf{X} 23$ & -0.2869705 & 0.12446353 & -2.305660 & $2.289116 \mathrm{e}-02$ \\
\hline
\end{tabular}

Table 9: Best Probable Subset LS Estimates - Dataset \# 2 
When we compare the fitted model to the population linear regression model, we can see that we obtained 4 out of the 5 regressor we used to generate the observations of $Y$, along with 3 nuisance variables as regressors. It is important to note here that not selecting every regressor having a non-zero regression coefficient is not necessarily a bad thing. It just means that the regressor did not contribute to the fitted model. To make proper conclusions, we compare the BPS method with other existing subset selection methods.

\begin{tabular}{|c|c|c|c|c|c|c|}
\hline & SubsetSize & BPS $\quad$ & BestSubset & Forward & Backward & Stepwise \\
\hline 1 & 1 & 0.4852 & 0.4852 & 0.4852 & 0.4852 & 0.4852 \\
\hline 2 & 2 & 0.5754 & 0.5754 & 0.5754 & 0.5754 & 0.5754 \\
\hline 3 & 3 & 0.6296 & 0.6296 & 0.6296 & 0.6296 & 0.6296 \\
\hline 4 & 4 & 0.6411 & 0.6411 & 0.6411 & 0.6392 & 0.6411 \\
\hline 5 & 5 & 0.6504 & 0.6504 & 0.6500 & 0.6504 & 0.6504 \\
\hline 6 & 6 & 0.6612 & 0.6612 & 0.6612 & 0.6612 & 0.6430 \\
\hline 7 & 7 & 0.6684 & 0.6684 & 0.6684 & 0.6684 & 0.6684 \\
\hline 8 & 8 & 0.6721 & 0.6749 & 0.6749 & 0.6749 & 0.6749 \\
\hline 9 & 9 & 0.6748 & 0.6790 & 0.6790 & 0.6790 & 0.6790 \\
\hline 10 & 10 & 0.6769 & 0.6834 & 0.6834 & 0.6824 & 0.6834 \\
\hline 11 & 11 & 0.6801 & 0.6876 & 0.6852 & 0.6876 & 0.6876 \\
\hline 12 & 12 & 0.6856 & 0.6894 & 0.6880 & 0.6894 & 0.6894 \\
\hline 13 & 13 & 0.6878 & 0.6897 & 0.6897 & 0.6897 & 0.6495 \\
\hline 14 & 14 & 0.6885 & 0.6899 & 0.6899 & 0.6899 & 0.6899 \\
\hline 15 & 15 & 0.6862 & 0.6896 & 0.6896 & 0.6896 & 0.6896 \\
\hline 16 & 16 & 0.6860 & 0.6894 & 0.6894 & 0.6894 & 0.6894 \\
\hline 17 & 17 & 0.6842 & 0.6886 & 0.6886 & 0.6886 & 0.6886 \\
\hline 18 & 18 & 0.6839 & 0.6876 & 0.6876 & 0.6876 & 0.6876 \\
\hline 19 & 19 & 0.6826 & 0.6857 & 0.6857 & 0.6857 & 0.6857 \\
\hline 20 & 20 & 0.6808 & 0.6836 & 0.6836 & 0.6836 & 0.6836 \\
\hline 21 & 21 & 0.6793 & 0.6814 & 0.6814 & 0.6814 & 0.6576 \\
\hline 22 & 22 & 0.6779 & 0.6786 & 0.6786 & 0.6786 & 0.6584 \\
\hline 23 & 23 & 0.6749 & 0.6758 & 0.6758 & 0.6758 & 0.6746 \\
\hline 24 & 24 & 0.6725 & 0.6728 & 0.6728 & 0.6728 & 0.6728 \\
\hline 25 & 25 & 0.6696 & 0.6696 & 0.6696 & 0.6696 & 0.6696 \\
\hline
\end{tabular}

Table 10: Subset Selection Methods $R_{a d j}^{2}$ Comparisons - Dataset \# 2 
Table 10 in the previous page contains information for comparing the different subset selection methods. The table provides the $R_{a d j}^{2}$ for each subset size using each subset selection method. The cells highlighted in yellow indicates the $R_{a d j}^{2}$ value for the best model selected by each method.

We can observe that the BPS method has the same $R_{a d j}^{2}$ values as most subset selection methods for all subset sizes. Most importantly, the $R_{a d j}^{2}$ values when the subset size equals 7 is the same for all subset selection methods and they all have the same regressors and LS estimates when $l=7$, so the BPS is performing just as well and reducing the dimensions even more than other methods. According to the subset selection criteria explained in chapter 1 , all pre-existing subset selection methods should choose $l=14$ as the best subset size, which is when $R_{a d j}^{2}$ reaches a maximum. The BPS method does not take any more regressors once the adjusted R-Squared value increases by less than $1 \%$, so we take $l=7$ regressors as the best probable subset size for the best probable model $M^{B P S}$.

We now compare the $M S E_{B P S}$ and $M S E_{R F}$. The table below provides a summary of the number of trees, number of regressors considered at each node, and MSE, for the Random Forest created for dataset \# 2:

\begin{tabular}{|l|r|}
\hline Measure & Value \\
\hline Number of Trees & 100.00000 \\
\hline Splits per Node & 8.00000 \\
\hline MSE & 53.37196 \\
\hline
\end{tabular}

Table 11: Random Forest Summary - Dataset \# 2

The $M S E_{R F}$ is much higher than the $M S E_{B P S}$, which equals 26.1753 , so we conclude that the BPS method yields lower variation than Random Forests for dataset \# 2. 


\section{Dataset \# 3:}

Number of Predictors: 50

Contributing Predictors: $X_{1}, X_{2}, \ldots, X_{10}$

Sample Size: 250

Error Term: $\varepsilon \sim N\left(0, \sigma^{2}=10\right)$

Maximum Pair-Wise Correlation Value: 0.205

Non-Zero Regression Coefficients:

\begin{tabular}{|c|c|c|}
\hline Regressors & Distribution & Coefficients \\
\hline$\times 1$ & X1 NORMAL $(M u=5$, Sigma $=10)$ & -2 \\
\hline $\mathrm{x} 2$ & X2 GAMMA(alpha $=6$, beta $=1)$ & -2 \\
\hline $\mathrm{x} 3$ & X3 GAMMA(alpha $=4$, beta $=5)$ & -6 \\
\hline $\mathrm{X} 4$ & X4 GAMMA(alpha $=10$, beta $=3)$ & 3 \\
\hline$\times 5$ & X5 GAMMA(alpha $=10$, beta $=1)$ & -8 \\
\hline $\mathrm{X} 6$ & X6 GAMMA(alpha $=9$, beta $=3)$ & 6 \\
\hline$x 7$ & X7 GAMMA(alpha $=10$, beta $=9)$ & 4 \\
\hline $\mathrm{x} 8$ & X8 GAMMA(alpha $=7$, beta $=2)$ & 1 \\
\hline$\times 9$ & X9 NORMAL $(M u=3$, Sigma $=4)$ & 10 \\
\hline $\mathrm{X} 10$ & X10 GAMMA(alpha $=3$, beta $=6)$ & -10 \\
\hline
\end{tabular}

Table 12: Regression Coefficients - Dataset \# 3

Just like in the two previous data set, any predictor variables that do not appear in the table above have a regression coefficient equal to zero. We generated 1000 models for each subset size $i$, where $1 \leq i \leq 50$, using $\mathrm{R}$. From each group of 1000 models, the best model of size $i$, denoted as $M_{i}^{B P S}$, is selected. The following plot and table show the adjusted Rsquares for each $M_{i}^{B P S}$ model. 


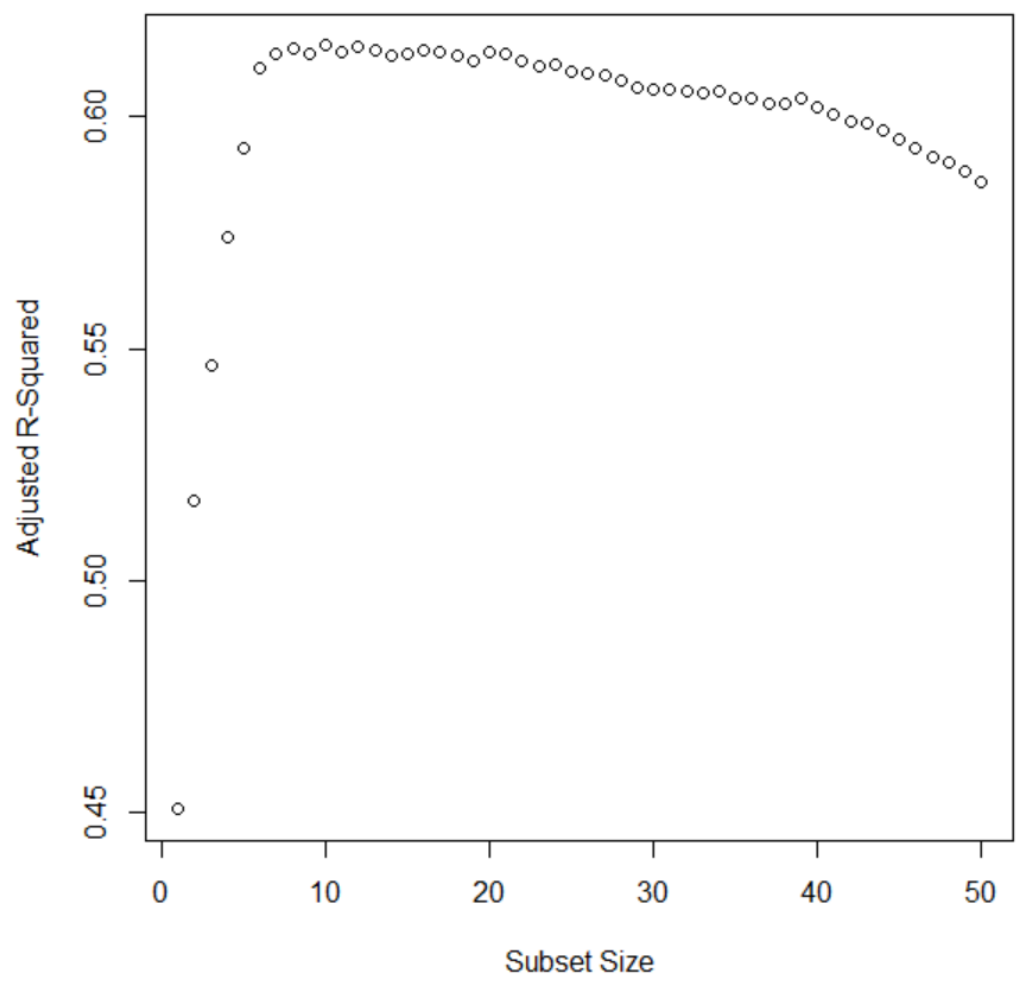

Figure 14: Adjusted R-Squares vs. Subset Size Plot-Dataset \# 3

\begin{tabular}{|c|c|c|c|c|c|}
\hline & SubsetSize & Regressors & RSquares & AdjustedRSquares & AdjRSquaresPercentChange \\
\hline 1 & 1 & 1 & 0.4529 & 0.4507 & NA \\
\hline 2 & 2 & 1,9 & 0.5209 & 0.5171 & 14.7 \\
\hline 3 & 3 & $1,9,5$ & 0.5519 & 0.5465 & 5.7 \\
\hline 4 & 4 & $9,1,5,4$ & 0.5810 & 0.5742 & 5.1 \\
\hline 5 & 5 & $1,5,4,2,9$ & 0.6013 & 0.5932 & 3.3 \\
\hline 6 & 6 & $1,4,5,9,45,2$ & 0.6201 & 0.6107 & 3.0 \\
\hline 7 & 7 & $1,9,45,5,2,46,4$ & 0.6247 & 0.6138 & 0.5 \\
\hline 8 & 8 & $26,1,45,9,13,5,4,2$ & 0.6270 & 0.6147 & 0.1 \\
\hline 9 & 9 & $1,27,2,32,5,28,9,4,45$ & 0.6276 & 0.6136 & -0.2 \\
\hline 10 & 10 & $1,9,4,39,28,45,5,34,43,2$ & 0.6309 & 0.6154 & 0.3 \\
\hline 11 & 11 & $1,2,4,9,20,22,45,5,26,6,13$ & 0.6313 & 0.6142 & -0.2 \\
\hline 12 & 12 & $1,9,4,39,28,45,5,34,43,2,31,46$ & 0.6336 & 0.6150 & 0.1 \\
\hline 13 & 13 & $1,45,5,13,9,2,4,31,34,26,43,10,46$ & 0.6346 & 0.6145 & -0.1 \\
\hline 14 & 14 & $1,45,5,13,9,2,4,31,34,26,43,10,46,15$ & 0.6351 & 0.6134 & -0.2 \\
\hline 15 & 15 & $1,45,15,9,5,13,2,49,25,34,31,26,29,46,4$ & 0.6371 & 0.6138 & 0.1 \\
\hline 16 & 16 & $1,45,15,9,5,13,2,49,25,34,31,26,29,46,4,39$ & 0.6392 & 0.6144 & 0.1 \\
\hline 17 & 17 & $1,15,32,31,4,39,45,2,26,5,16,9,13,14,34, \ldots$ & 0.6405 & 0.6141 & 0.0 \\
\hline 18 & 18 & $1,4,10,13,5,12,45,9,39,2,34,14,15,20,31, \ldots$ & 0.6411 & 0.6131 & -0.2 \\
\hline 19 & 19 & $1,4,10,13,5,12,45,9,39,2,34,14,15,20,31, \ldots$ & 0.6416 & 0.6120 & -0.2 \\
\hline 20 & 20 & $9,1,43,4,45,20,13,2,15,25,17,34,28,10,31, \ldots$ & 0.6451 & 0.6141 & 0.3 \\
\hline
\end{tabular}

Table 13: Best Probable Subset of Size l-Dataset \# 3 
Note that the table above does not contain the entire list of best probable models for all sizes. Rather, the list contains the best probable models for subset sizes 1 to 20 . The rest are omitted as the entire table takes a lot of space and we are only concerned with the first seven rows. Regardless, according to our stopping rule, we should select the best probable subset of six regressors, $X_{1}, X_{2}, X_{4}, X_{5}, X_{9}$, and $X_{45}$, which yield the following LS estimates:

\begin{tabular}{|r|r|l|r|l|} 
& Estimate & Std. Error & \multicolumn{1}{|l|}{ t value } & $\operatorname{Pr}(>|\mathrm{t}|)$ \\
\hline (Intercept) & 13.4702129 & 3.81414217 & 3.531649 & $4.940188 \mathrm{e}-04$ \\
\hline $\mathbf{X 1}$ & 0.9715304 & 0.05762831 & 16.858563 & $9.362526 \mathrm{e}-43$ \\
\hline X2 & 0.9327886 & 0.24894296 & 3.746997 & $2.236171 \mathrm{e}-04$ \\
\hline X4 & 2.5726850 & 0.58762093 & 4.378137 & $1.779389 \mathrm{e}-05$ \\
\hline X5 & 0.8885533 & 0.20209862 & 4.396632 & $1.644650 \mathrm{e}-05$ \\
\hline X9 & 1.0331887 & 0.16404468 & 6.298215 & $1.399570 \mathrm{e}-09$ \\
\hline X45 & -4.7786485 & 1.37897278 & -3.465368 & $6.258485 \mathrm{e}-04$ \\
\hline
\end{tabular}

Table 14: Best Probable Subset LS Estimates - Dataset \# 3

When we compare the fitted model to the population linear regression model, we can see that we obtained 5 out of the 10 regressor we used to generate the observations of $Y$, along with only one predictor variable $\left(X_{45}\right)$ not in the original population model. Let us now see how the BPS method performed compared to other subset selection methods. The table in the next page contains the $R_{a d j}^{2}$ for each subset size selected by each subset selection method. Note from the table that only the first 20 best probable subset sizes are included. We can see that the $R_{a d j}^{2}$ value for a subset selection of size $l=6$ is equal for all subset selection methods, including BPS. 


\begin{tabular}{|c|c|c|c|c|c|c|}
\hline & SubsetSizê & BPS & BestSubset & Forward & Backward & Stepwise \\
\hline 1 & 1 & 0.4507 & 0.4507 & 0.4507 & 0.4507 & 0.4507 \\
\hline 2 & 2 & 0.5171 & 0.5171 & 0.5171 & 0.5171 & 0.5171 \\
\hline 3 & 3 & 0.5465 & 0.5465 & 0.5465 & 0.5465 & 0.5465 \\
\hline 4 & 4 & 0.5742 & 0.5742 & 0.5742 & 0.5742 & 0.5742 \\
\hline 5 & 5 & 0.5932 & 0.5932 & 0.5932 & 0.5932 & 0.5300 \\
\hline 6 & 6 & 0.6107 & 0.6107 & 0.6107 & 0.6107 & 0.6107 \\
\hline 7 & 7 & 0.6138 & 0.6148 & 0.6148 & 0.6139 & 0.6148 \\
\hline 8 & 8 & 0.6147 & 0.6186 & 0.6183 & 0.6182 & 0.6186 \\
\hline 9 & 9 & 0.6136 & 0.6220 & 0.6216 & 0.6220 & 0.6220 \\
\hline 10 & 10 & 0.6154 & 0.6264 & 0.6242 & 0.6264 & 0.5926 \\
\hline 11 & 11 & 0.6142 & 0.6283 & 0.6278 & 0.6283 & 0.6283 \\
\hline 12 & 12 & 0.6150 & 0.6297 & 0.6297 & 0.6297 & 0.6297 \\
\hline 13 & 13 & 0.6145 & 0.6315 & 0.6315 & 0.6315 & 0.6315 \\
\hline 14 & 14 & 0.6134 & 0.6322 & 0.6322 & 0.6322 & 0.6322 \\
\hline 15 & 15 & 0.6138 & 0.6327 & 0.6327 & 0.6327 & 0.6327 \\
\hline 16 & 16 & 0.6144 & 0.6331 & 0.6329 & 0.6331 & 0.6331 \\
\hline 17 & 17 & 0.6141 & 0.6330 & 0.6328 & 0.6330 & 0.5930 \\
\hline 18 & 18 & 0.6131 & 0.6329 & 0.6329 & 0.6329 & 0.6329 \\
\hline 19 & 19 & 0.6120 & 0.6323 & 0.6323 & 0.6323 & 0.6323 \\
\hline 20 & 20 & 0.6141 & 0.6319 & 0.6319 & 0.6319 & 0.5900 \\
\hline
\end{tabular}

Table 15: Subset Selection Methods $R_{a d j}^{2}$ Comparisons - Dataset \# 3

We have equal adjusted $R^{2}$ s because all methods selected the same regressors for a subset of size $l=6$. We can see from the table that the other subset selection methods also selected some predictors that had a regression parameter equal to zero in the true population model. Note also that the BPS method selected a smaller subset of regressors which yields a very similar $R_{a d j}^{2}$ value to the subset selected by the other subset selection methods. When we compare the BPS method to the best Best Subset Selection method, we can see that there is a relatively large difference in the number of regressors selected for the fitted model while the difference in their $R_{a d j}^{2}$ is very small. The fitted model using Best Subset Selection has 16 regressors and an $R_{a d j}^{2}$ of 0.6331 while the BPS gives us a model with only 6 regressors and an $R_{a d j}^{2}$ of 0.617 - a small difference in $R_{a d j}^{2}$ for a large difference 
in number of regressors, which is very useful in high dimensional data. Lastly, the reduced model we get using BPS yields an R-squared value of 0.6201 , which is very close to the full regression R-squared, which is 0.6692 . The table below provides all R-Squared values for each subset obtained using each subset selection method.

\begin{tabular}{|c|c|c|c|c|c|c|}
\hline & SubsetSize & BPS $\approx$ & BestSubset & Forward & Backward & Stepwise \\
\hline 1 & 1 & 0.4529 & 0.4529 & 0.4529 & 0.4529 & 0.4529 \\
\hline 2 & 2 & 0.5209 & 0.5209 & 0.5209 & 0.5209 & 0.5209 \\
\hline 3 & 3 & 0.5519 & 0.5519 & 0.5519 & 0.5519 & 0.5519 \\
\hline 4 & 4 & 0.5810 & 0.5810 & 0.5810 & 0.5810 & 0.5810 \\
\hline 5 & 5 & 0.6013 & 0.6013 & 0.6013 & 0.6013 & 0.5394 \\
\hline 6 & 6 & 0.6201 & 0.6201 & 0.6201 & 0.6201 & 0.6201 \\
\hline 7 & 7 & 0.6247 & 0.6256 & 0.6256 & 0.6248 & 0.6256 \\
\hline 8 & 8 & 0.6270 & 0.6308 & 0.6305 & 0.6305 & 0.6308 \\
\hline 9 & 9 & 0.6276 & 0.6357 & 0.6353 & 0.6357 & 0.6357 \\
\hline 10 & 10 & 0.6309 & 0.6414 & 0.6393 & 0.6414 & 0.6090 \\
\hline 11 & 11 & 0.6313 & 0.6447 & 0.6442 & 0.6447 & 0.6447 \\
\hline 12 & 12 & 0.6336 & 0.6475 & 0.6475 & 0.6475 & 0.6475 \\
\hline 13 & 13 & 0.6346 & 0.6507 & 0.6507 & 0.6507 & 0.6507 \\
\hline 14 & 14 & 0.6351 & 0.6529 & 0.6529 & 0.6529 & 0.6529 \\
\hline 15 & 15 & 0.6371 & 0.6548 & 0.6548 & 0.6548 & 0.6548 \\
\hline 16 & 16 & 0.6392 & 0.6566 & 0.6564 & 0.6566 & 0.6566 \\
\hline 17 & 17 & 0.6405 & 0.6580 & 0.6579 & 0.6580 & 0.6208 \\
\hline 18 & 18 & 0.6411 & 0.6594 & 0.6594 & 0.6594 & 0.6594 \\
\hline 19 & 19 & 0.6416 & 0.6604 & 0.6604 & 0.6604 & 0.6604 \\
\hline 20 & 20 & 0.6451 & 0.6614 & 0.6614 & 0.6614 & 0.6229 \\
\hline
\end{tabular}

Table 16: Subset Selection Methods $R^{2}$ Comparisons - Dataset \# 3

Finally, the $M S E_{B P S}$ we obtained for dataset \# 3 equals 99.6679, a much lower value than the $M S E_{R F}$, which is provided in the table below:

\begin{tabular}{|l|r|}
\hline Measure & Value \\
\hline Number of Trees & 100.0000 \\
\hline Splits per Node & 17.0000 \\
\hline MSE & 158.1862 \\
\hline
\end{tabular}

Table 17: Random Forest Summary - Dataset \# 3 


\section{Dataset \# 4:}

Number of Predictors: 100

Contributing Predictors: $X_{1}, X_{2}, \ldots, X_{20}$

Sample Size: 80

Error Term: $\varepsilon \sim N\left(0, \sigma^{2}=10\right)$

Maximum Pair-Wise Correlation Value: 0.440

Non-Zero Regression Coefficients:

\begin{tabular}{|c|c|c|}
\hline Regressors & Distribution & Coefficients \\
\hline $\mathrm{X} 1$ & XI GAMMA(alpha $=5$, beta $=5)$ & -10 \\
\hline $\mathrm{x} 2$ & $\mathrm{X} 2$ GAMMA $($ alpha $=8$, beta $=1)$ & -10 \\
\hline $\mathrm{X} 3$ & X3 GAMMA(alpha $=6$, beta $=2)$ & -4 \\
\hline $\mathrm{X} 4$ & X4 CHI-SQUARED $(\mathrm{df}=8)$ & 8 \\
\hline X5 & X5 NORMAL $(M u=7$, Sigma $=7)$ & 2 \\
\hline $\mathrm{x} 6$ & X6 NORMAL $(M u=9$, Sigma $=3)$ & -10 \\
\hline $\mathrm{X} 7$ & X7 GAMMA(alpha $=9$, beta $=3)$ & 7 \\
\hline $\mathrm{x} 8$ & X8 NORMAL $(M u=1, \operatorname{sigma}=7)$ & 8 \\
\hline$x 9$ & X9 NORMAL $(M u=7$, Sigma $=2)$ & -2 \\
\hline$\times 10$ & X10 CHI-SQUARED $(\mathrm{df}=6)$ & -5 \\
\hline$\times 11$ & $\mathrm{X} 11 \mathrm{CHI}-\mathrm{SQUARED}(\mathrm{df}=3)$ & -10 \\
\hline $\mathrm{X} 12$ & X12 GAMMA $($ alpha $=2$, beta $=10)$ & -7 \\
\hline $\mathrm{X} 13$ & X13 GAMMA (alpha $=2$, beta $=2)$ & 5 \\
\hline$\times 14$ & X14 NORMAL $(M u=2$, Sigma $=2)$ & 7 \\
\hline$\times 15$ & X15 GAMMA(alpha $=5$, beta $=3)$ & -8 \\
\hline $\mathrm{X} 16$ & X16 NORMAL $(M u=5$, Sigma $=9)$ & -7 \\
\hline$\times 17$ & X17 GAMMA(alpha $=4$, beta $=9)$ & 7 \\
\hline $\mathrm{X} 18$ & X18 GAMMA $($ alpha $=8$, beta $=10)$ & -10 \\
\hline $\mathrm{X} 19$ & X19 GAMMA(alpha $=3$, beta $=9)$ & -1 \\
\hline $\mathrm{X} 20$ & X20 GAMMA(alpha $=10$, beta $=9)$ & 7 \\
\hline
\end{tabular}

Table 18: Regression Coefficients - Dataset \# 4 
Note that, unlike the previous datasets, dataset \# 4 has high dimensionality, with a sample size of 80 and 100 predictor variables. The table above shows the predictor variables that contributed to the simulation of the response variable $Y$, along with their respective distributions and regression coefficients; any predictor variables that do not appear in the table above have a regression coefficient equal to zero. We generated 1000 models for each subset size $i$, where $1 \leq i \leq 80 / 5$, using R. Recall that one of our stopping rules was that the sample size $n$ has to be at least five times the subset size, or the number of regressors in the reduced model. Since we have 80 observations, we will only look at the best probable subsets of sizes 1 through $\frac{80}{5}=16$. The following plot and table show the adjusted Rsquares for each $M_{i}^{B P S}$ model.

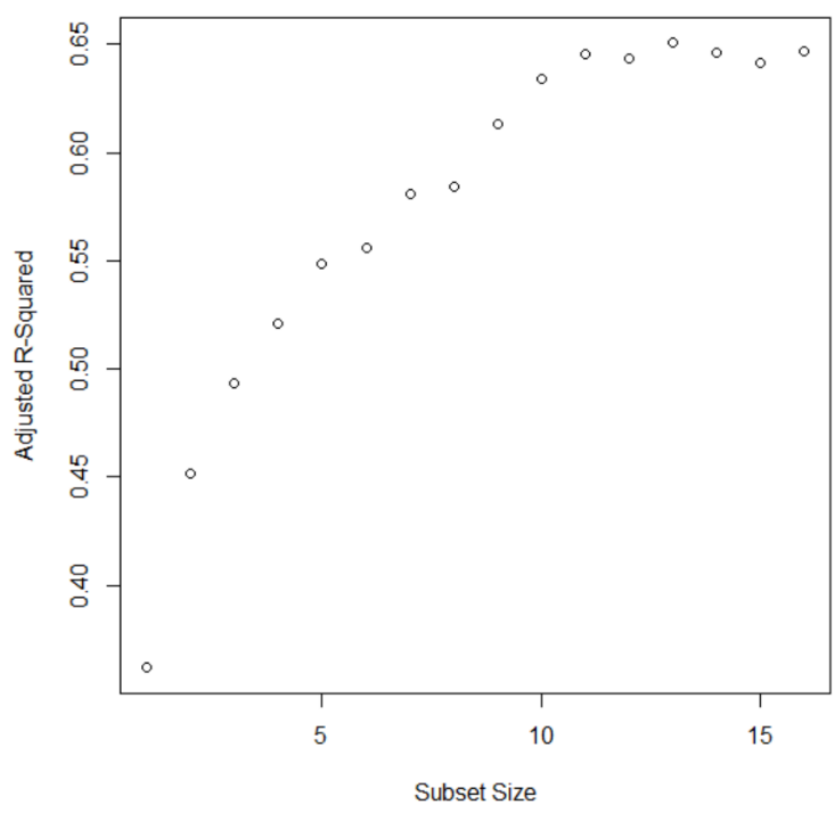

Figure 15: Adjusted R-Squares vs. Subset Size Plot - Dataset \# 4 


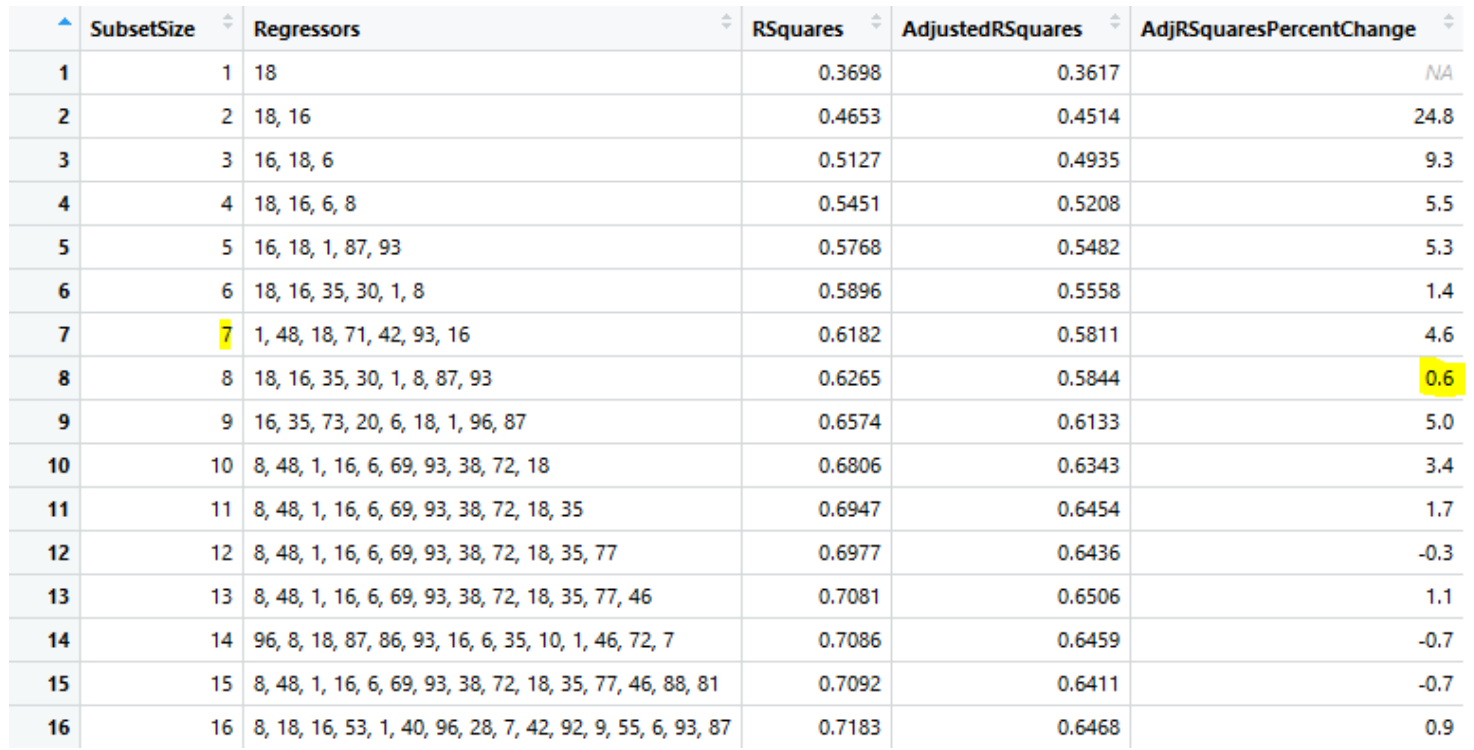

Table 19: Best Probable Subset of Size l - Dataset \# 4

The table above contains the best probable models for subset sizes 1 through 16 . Regardless, according to our stopping rule, we should select the best probable subset of seven regressors, $X_{1}, X_{16}, X_{18}, X_{42}, X_{71}$, and $X_{93}$, which yield the following LS estimates:

\begin{tabular}{|r|r|l|l|l|}
\hline & Estimate & $\begin{array}{l}\text { Std. } \\
\text { Error }\end{array}$ & t value & $\operatorname{Pr}(>|\mathbf{t}|)$ \\
\hline Intercept) & 59.6013084 & 8.6613770 & 6.8812740 & $1.828538 \mathrm{e}-09$ \\
\hline $\mathbf{X 1}$ & 17.2286157 & 6.2860116 & 2.7407865 & $7.724348 \mathrm{e}-03$ \\
\hline $\mathbf{X 1 6}$ & 1.3579303 & 0.3904026 & 3.4782817 & $8.601199 \mathrm{e}-04$ \\
\hline $\mathbf{X 1 8}$ & 1.3692709 & 0.1737808 & 7.8792976 & $2.575598 \mathrm{e}-11$ \\
\hline $\mathbf{X} 42$ & -1.1897033 & 1.9487501 & -0.6104955 & $5.434559 \mathrm{e}-01$ \\
\hline $\mathbf{X} 48$ & -7.2099514 & 2.5908994 & -2.7827987 & $6.877467 \mathrm{e}-03$ \\
\hline $\mathbf{X 7 1}$ & 7.6710046 & 3.2643305 & 2.3499472 & $2.152067 \mathrm{e}-02$ \\
\hline $\mathbf{X 9 3}$ & 0.7579273 & 0.2452512 & 3.0904118 & $2.841155 \mathrm{e}-03$ \\
\hline
\end{tabular}

Table 20: Best Probable Subset LS Estimates - Dataset \# 4

When we compare the fitted model to the population linear regression model, we can see that we obtained 3 out of the 20 regressor we used to generate the observations of $Y$, along with four other predictor variables. Let us now see how the BPS method performed 
compared to forward selection. The following table contains the $R_{a d j}^{2}$ for each subset size selected by each subset selection method:

\begin{tabular}{|c|c|c|c|}
\hline $\boldsymbol{\Delta}$ & SubsetSize & BPS & Forward \\
\hline 1 & 1 & 0.3617 & 0.3617 \\
\hline 2 & 2 & 0.4514 & 0.4514 \\
\hline 3 & 3 & 0.4935 & 0.4954 \\
\hline 4 & 4 & 0.5208 & 0.5319 \\
\hline 5 & 5 & 0.5482 & 0.5635 \\
\hline 6 & 6 & 0.5558 & 0.5930 \\
\hline 7 & 7 & 0.5811 & 0.6219 \\
\hline 8 & 8 & 0.5844 & 0.6504 \\
\hline 9 & 9 & 0.6133 & 0.6789 \\
\hline 10 & 10 & 0.6343 & 0.7017 \\
\hline 11 & 11 & 0.6454 & 0.7136 \\
\hline 12 & 12 & 0.6436 & 0.7211 \\
\hline 13 & 13 & 0.6506 & 0.7282 \\
\hline 14 & 14 & 0.6459 & 0.7378 \\
\hline 15 & 15 & 0.6411 & 0.7515 \\
\hline 16 & 16 & 0.6468 & 0.7588 \\
\hline
\end{tabular}

Table 21: Subset Selection Methods $R_{\text {adj }}^{2}$ Comparisons - Dataset \# 4

Note from the table above that we are only comparing the BPS method with the Forward Selection method. The other methods cannot be used with data that has high dimensionality [3]. We can see that the $R_{a d j}^{2}$ value for a subset selection of size $l=7$ using the BPS method is slightly lower than the subset of the same size obtained using forward selection. When comparing the BPS to random forests, we see that the $M S E_{B P S}$, which equals 209.369 , is once again much lower than the $M S E_{R F}$, provided in the table below.

\begin{tabular}{|l|r|} 
Measure & Value \\
\hline Number of Trees & 100.0000 \\
\hline Splits per Node & 33.0000 \\
\hline MSE & 370.7178 \\
\hline
\end{tabular}

Table 22: Random Forest Summary - Dataset \# 4 


\section{Dataset \# 5: College Dataset}

Number of Predictors: 16

Sample Size: 777

Important Information:

The College dataset selected for this section was taken from the ISLR package in R. The dataset contains a total of 18 variables. From these, we have selected the Graduation Rates, Grad.Rate, as the response variable. We also dropped out the Private variable because it is non-numeric, and it would interfere with the correlation matrix. The remaining 16 variables were used as predictor variables. We now use various methods to select a subset of the predictor variables to fit a model for the graduation rates, which we have renamed as $Y$ in the dataset.

Model Assumptions:

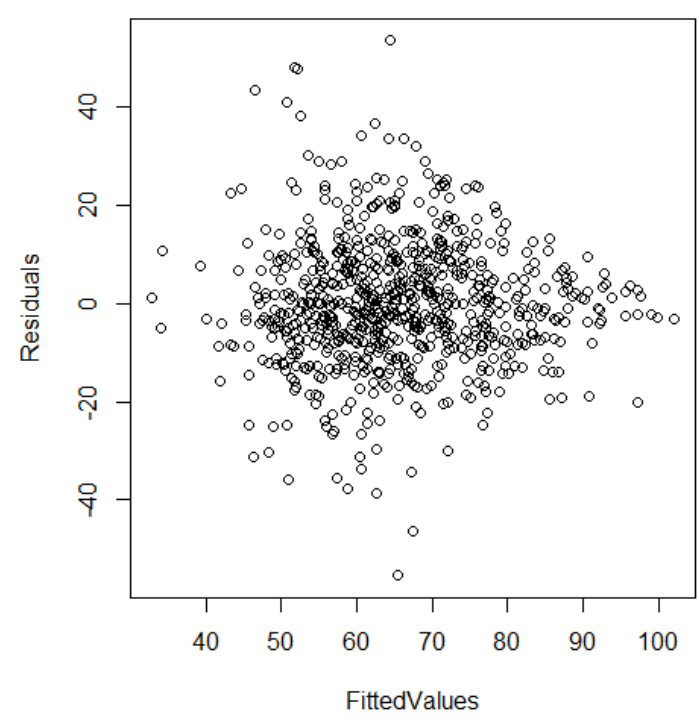

Figure 16: Residuals vs. Fitted Values - Dataset \# 5

The Fitted Values vs. Residuals plot shown above shows no pattern of the data, so we can assume that the response variable is linear and the error terms have a constant variance. 
The correlation matrix, however, shows very high values amongst many pairs of regressors; the correlation values go as high as 0.943 between the variables Apps and Accept, which are the number of applications received and number of applications accepted, respectively. Regardless of the collinearity, we continue by selecting the best probable model using the BPS method.

Note that the sample size is very large compared to the number of predictor variables. Since we do not know the true model for the College dataset, we use cross validation. $80 \%$ of the observations were randomly selected for the train set and the remaining $20 \%$ of the observations were used for the test set. The "random" selection of observations was done in R, using set. seed(1) and sample() to generate the random indexes for the observations that belong to the train data set. We proceed with multiple subset selection methods to find out which subset of predictors can contribute to the fitted model. The following plot and table show the adjusted R-squares for each $M_{i}^{B P S}$ model.

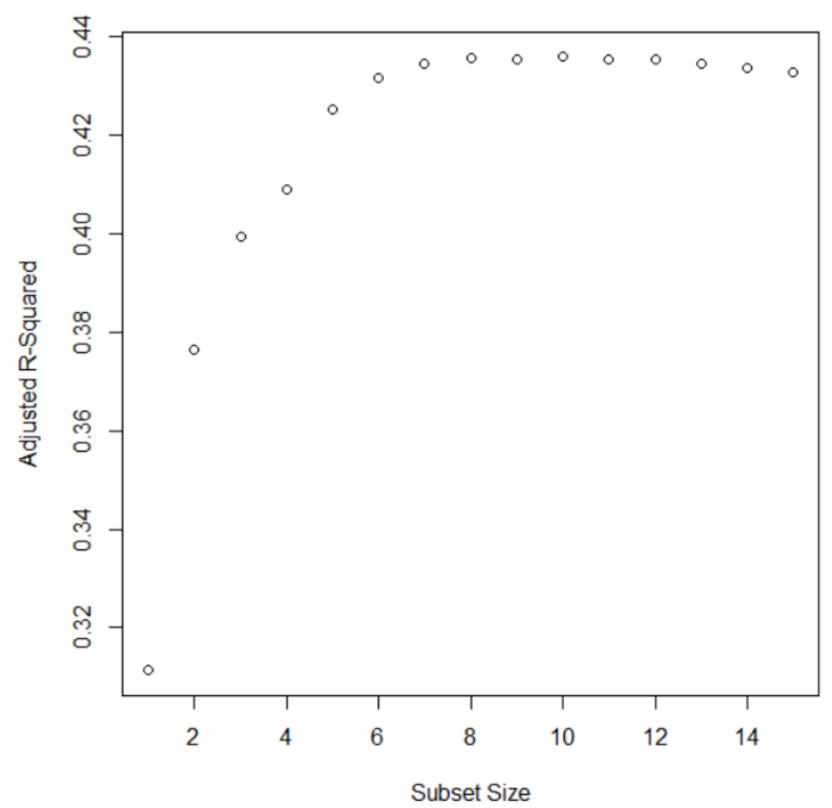

Figure 17: Adjusted R-Squares vs. Subset Size Plot-Dataset \# 5 


\begin{tabular}{|c|c|c|c|c|c|}
\hline & SubsetSizê & Regressors & RSquares & AdjustedRSquares & AdjRSquaresPercentChange \\
\hline 1 & 1 & 8 & 0.3125 & 0.3113 & NA \\
\hline 2 & 2 & 8,5 & 0.3784 & 0.3764 & 20.9 \\
\hline 3 & 3 & $15,5,8$ & 0.4023 & 0.3994 & 6.1 \\
\hline 4 & 4 & $7,5,15,8$ & 0.4127 & 0.4089 & 2.4 \\
\hline 5 & 5 & $15,8,1,7,5$ & 0.4299 & 0.4253 & 4.0 \\
\hline 6 & 6 & $7,8,15,5,9,1$ & 0.4371 & 0.4316 & 1.5 \\
\hline 7 & 7 & $8,5,15,1,9,11,7$ & 0.4410 & 0.4346 & 0.7 \\
\hline 8 & 8 & $8,5,15,1,9,11,7,14$ & 0.4429 & 0.4356 & 0.2 \\
\hline 9 & 9 & $14,9,4,5,1,8,15,7,11$ & 0.4434 & 0.4352 & -0.1 \\
\hline 10 & 10 & $12,9,14,5,15,13,8,7,1,11$ & 0.4449 & 0.4358 & 0.1 \\
\hline 11 & 11 & $13,1,15,9,8,11,7,12,5,4,14$ & 0.4453 & 0.4353 & -0.1 \\
\hline 12 & 12 & $8,7,5,13,1,4,15,9,11,14,12,6$ & 0.4462 & 0.4352 & 0.0 \\
\hline 13 & 13 & $8,12,15,5,9,13,4,7,14,11,1,3,6$ & 0.4463 & 0.4345 & -0.2 \\
\hline 14 & 14 & $15,13,1,4,7,11,8,5,14,9,2,12,6,10$ & 0.4464 & 0.4336 & -0.2 \\
\hline 15 & 15 & $13,1,15,9,8,11,7,12,5,4,14,6,2,3,10$ & 0.4465 & 0.4328 & -0.2 \\
\hline
\end{tabular}

Table 23: Best Probable Subset of Size l-Dataset \# 5

Note that the regressors have a number rather than a name. The table below matches each predictor number with its name:

\begin{tabular}{|c|c|}
\hline Number $\hat{\quad}$ & Name $\quad \hat{v}$ \\
\hline 1 & Apps \\
\hline 2 & Accept \\
\hline 3 & Enroll \\
\hline 4 & Top10perc \\
\hline 5 & Top25perc \\
\hline 6 & F.Undergrad \\
\hline 7 & P.Undergrad \\
\hline 8 & Outstate \\
\hline 9 & Room.Board \\
\hline 10 & Books \\
\hline 11 & Personal \\
\hline 12 & $\mathrm{PhD}$ \\
\hline 13 & Terminal \\
\hline 14 & S.F.Ratio \\
\hline 15 & perc.alumni \\
\hline 16 & Expend \\
\hline
\end{tabular}

Table 24: Regressor Numbers and Names - Dataset \# 5 
According to the BPS method, the best probable subset has seven predictors: Apps, Top25perc, P.Undergrad, Outstate, Room.Board, perc.alumni, and Expend. These seven predictors yield an R-squared of 0.4482, almost as large as the full model's R-Squared, which is 0.4587 . The reduced model has the following LS estimates:

\begin{tabular}{|r|l|l|l|l|} 
& Estimate & Std. Error & t value & $\operatorname{Pr}(>|\mathrm{t}|)$ \\
\hline (Intercept) & 31.0043040778 & 2.4340721998 & 12.737627 & $3.825139 \mathrm{e}-33$ \\
\hline P.Undergrad & -0.0022920695 & 0.0004529436 & -5.060386 & $5.535644 \mathrm{e}-07$ \\
\hline Outstate & 0.0009826236 & 0.0002090570 & 4.700267 & $3.210061 \mathrm{e}-06$ \\
\hline perc.alumni & 0.2757214343 & 0.0547289967 & 5.037941 & $6.196553 \mathrm{e}-07$ \\
\hline Top25perc & 0.1760505711 & 0.0333378192 & 5.280806 & $1.787701 \mathrm{e}-07$ \\
\hline Room.Board & 0.0017651253 & 0.0006312152 & 2.796392 & $5.329445 \mathrm{e}-03$ \\
\hline Apps & 0.0006345242 & 0.0001581418 & 4.012375 & $6.753096 \mathrm{e}-05$ \\
\hline
\end{tabular}

Table 25: Best Probable Subset LS Estimates - Dataset \# 5

Note that from the two regressors that had the highest pair-wise correlation (Apps and Accept), only Apps made it into the reduced model. The table in the next page contains the $R_{a d j}^{2}$ for each subset size selected by each subset selection method.

Note that the BPS selected the smallest subset out of all the subset selection methods, with a slightly smaller $R_{a d j}^{2}$. We can see that the $R_{a d j}^{2}$ value for a subset selection of size $l=7$ is the same for all methods. This is because all methods chose the same predictor variables when the subset size equals 7. 


\begin{tabular}{|r|r|r|r|r|r|}
\hline SubsetSize & BPS & BestSubset & Forward & Backward & Stepwise \\
\hline 1 & 0.3113 & 0.3113 & 0.3113 & 0.3113 & 0.3113 \\
\hline 2 & 0.3764 & 0.3764 & 0.3764 & 0.3764 & 0.3764 \\
\hline 3 & 0.3994 & 0.3994 & 0.3994 & 0.3994 & 0.3994 \\
\hline 4 & 0.4089 & 0.4089 & 0.4089 & 0.4089 & 0.4089 \\
\hline 5 & 0.4253 & 0.4253 & 0.4253 & 0.4253 & 0.2968 \\
\hline 6 & 0.4316 & 0.4316 & 0.4316 & 0.4316 & 0.4316 \\
\hline 7 & 0.4346 & 0.4346 & 0.4346 & 0.4346 & 0.4346 \\
\hline 8 & 0.4356 & 0.4356 & 0.4356 & 0.4356 & 0.4356 \\
\hline 9 & 0.4352 & 0.4357 & 0.4357 & 0.4352 & 0.4357 \\
\hline 10 & 0.4358 & 0.4358 & 0.4354 & 0.4358 & 0.4354 \\
\hline 11 & 0.4353 & 0.4357 & 0.4348 & 0.4357 & 0.4348 \\
\hline 12 & 0.4352 & 0.4352 & 0.4341 & 0.4352 & 0.4352 \\
\hline 13 & 0.4345 & 0.4345 & 0.4345 & 0.4345 & 0.4345 \\
\hline 14 & 0.4336 & 0.4337 & 0.4337 & 0.4337 & 0.4131 \\
\hline 15 & 0.4328 & 0.4328 & 0.4328 & 0.4328 & 0.4328 \\
\hline & & & & & \\
\hline
\end{tabular}

Table 26: Subset Selection Methods $R_{a d j}^{2}$ Comparisons - Dataset \# 5

When comparing the BPS to random forests, we see that the $M S E_{B P S}$, which equals 173.3057 , is somewhat lower than the $M S E_{R F}$, provided in the table below.

\begin{tabular}{l|r|}
\hline Measure & Value \\
\hline Number of Trees & 100.0000 \\
\hline Splits per Node & 5.0000 \\
\hline MSE & 178.4219 \\
\hline
\end{tabular}

Table 27: Random Forest Summary - Dataset \# 5

Note that both $M S E_{B P S}$ and $M S E_{R F}$ are calculated using the predictions from the test data set using cross validation.

Here we conclude the analysis and comparison of the Best Probable Subset and other subset selection methods with simulated or pre-existing datasets. 


\section{CHAPTER V}

\section{CONCLUSIONS AND REMARKS}

In this paper, we have discussed some concepts in Linear Regression, including the Least Squares Estimators (LSE), model assumptions, measures of fit, and subset selection. We also discussed some of the predominant methods used in linear regression for subset selection and the concept of regression trees and random forests, some of which can be used when dealing with high dimensional data.

We continued our discussion by proposing a new subset selection method, which we called Best Probable Subset (BPS), that seems to have great potential based on our simulations and can be used when we are dealing with high dimensional data. The Best Probable Subset (BPS) method is summarized here:

1. Create a probability pool, called Linear Relationship Pool (LRP), for selecting a subset of regressors by using the following formula for each regressor of the $k$ regressors.

$$
p_{j}=P\left(X_{j}\right)=\frac{r_{j}^{2}}{\sum_{i=1}^{k} r_{i}^{2}}, 1 \leq j \leq k
$$

Where $p_{j}$ is the probability of choosing the $j^{\text {th }}$ predictor for the reduced model and $r_{j}^{2}$ represents the squared correlation between the $j^{\text {th }}$ predictor and the response variable.

2. Begin by finding the null model $M_{0}^{B P S}$. For $1 \leq i \leq \min \left(k, \frac{n}{5}\right)$ :

a. Select a subset of size $i$ without replacement from the predictor by using the LRP. 
b. Continue finding the model with the best possible subset size $i$ until $M_{i}^{B P S}$ yields an $R^{2}{ }_{a d j}$ than is less than $1 \%$ greater than the $R^{2}$ adj of $M_{i-1}^{B P S}$.

3. Since the adjusted R-squared continues to increase by more than $1 \%$ until $M_{i-1}^{B P S}$, the best probable model is $M^{B P S}=M_{i-1}^{B P S}$.

We used the BPS method on multiple simulated dataset and obtained promising results when compared to Best Subset Selection and other subset selection methods by keeping up with an equal $R_{a d j}^{2}$ in the $1^{\text {st }}, 2^{\text {nd }}, 3^{\text {rd }}$, and $5^{\text {th }}$ datasets and a slightly lower $R_{a d j}^{2}$ on the $4^{\text {th }}$ dataset. The method was also compared with random forests and outperformed it with a lower MSE in every single dataset.

For future research projects, we would like to do further research on the theoretical side of the BPS method, which seems to have potential with low and high dimensional data. Without showing the theoretical proof, we can guarantee that the best probable subset will choose the best subset of size $l$ as the number of model selection repetitions (1000 in this paper) increase. A further research on this subset could include this proof. We would also like to investigate subset selection based on some other alternative subset selection probability rules. A way to improve this study would be to test the method with many more datasets of different sizes and properties and see how it performs compared to other subset selection methods. Furthermore, for 1000 models with a subset size $l$, we could select the model that yields the lowest MSE instead of the highest $R^{2}$. More research can be done on the performance of the method overall by finding the average proportion of the $R^{2}$ of the full model that is explained by the $R^{2}$ of the best probable model throughout many datasets. 


\section{REFERENCES}

1. Montgomery, Douglas C., Elizabeth A. Peck, and Geoffrey Vining. Introduction to Linear Regression Analysis. 5th ed. Hoboken, NJ: Wiley, 2012.

2. Mandenhall, William. Mathematical Statistics with Applications. 7th ed. Boston, MA: Thomson Brooks/Cole, 2008.

3. James, Gareth, Daniela Witten, Trevor Hastie, and Robert Tibshirani. An Introduction to Statistical Learning with Applications in R. 1st ed. New York, NY: Springer, 2013.

4. Hocking, Ronald R. Methods and Applications of Linear Models: Regression and the Analysis of Variance. 2nd ed. Hoboken, NJ: John Wiley and Sons, 2003.

5. Rohatgi, Vijay K. An Introduction to Probability and Statistics. 2nd ed. New York, NY: John Wiley and Sons, 2001.

6. Walpole, Ronald E. Probability and Statistics for Engineers and Scientists. 6th ed. New Jersey, NJ: Prentice-Hall, 2002.

7. Lyman, R. Ott. An Introduction to Statistical Methods and Data Analysis. 6th ed. Bermont, CA: Cengage, 2008.

8. Rawlings, John O. Applied Regression Analysis: A Research Tool. 1st ed. New York, NY: Springer, 1988.

9. Kotu, Vijay. Predictive Analytics and Data Mining. Waltham, MA: Elsevier, 2015.

10. Hastie, Trevor. The Elements of Statistical Learning. 2nd ed. Stanford, CA: Springer, 2016.

11. James, Gareth, Daniela Witten, Trevor Hastie, and Rob Tibshirani. "Package 'ISLR'." R-Project. October 19, 2017. https://cran.rproject.org/web/packages/ISLR/ISLR.pdf.

12. Strobl, Carolin, Anne-Laure Boulesteix, Thomas Kneib, Thomas Augustin, and Achim Zeileis. "Conditional Variable Importance for Random Forests." BMC Bioinformatics9, no. 1 (2008). doi:10.1186/1471-2105-9-307.

13. Genuer, Robin, Jean-Michel Poggi, and Christine Tuleau-Malot. "Variable Selection Using Random Forests." Pattern Recognition Letters31, no. 14 (2010): 2225-236. doi:10.1016/j.patrec.2010.03.014. 


\section{APPENDIX}

\section{APPENDIX A}

\section{Program R-Code}

The following code can be copied and pasted into R-Studio. This code will simulate data and do all the analysis explained throughout the entirety of this paper. To avoid running errors caused by different versions, it is advised to use Microsoft R Open 3.5.1 and RStudio 1.0.143, which are the versions I used when writing the program. Without further ado, here is the R-Code:

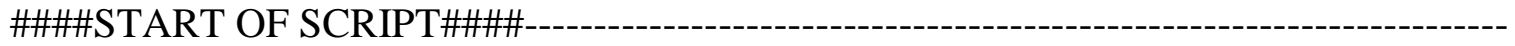
\#\#\#\#\#\#MASTER'S THESIS - ELIESER NODARSE\#\#\#\#\#
\#\#\#\#THE PURPOSE OF THIS PROGRAM IS TO ANALYZE THE IMPACT OF
\#\#\#\#DATA REDUCTION IN LINEAR REGRESSION

\#\#\#\#INSTALL NECESSARY PACKAGES\#\#\#\#

install.packages(c("dplyr",

"stringr",

"DT",

"MASS",

"gtools",

"ISLR",

"leaps",

"glmnet",

"quantmod"

))

library(dplyr)

library(stringr)

library(DT)

library(MASS)

library(gtools)

library(ISLR)

library(leaps)

library(glmnet)

library(quantmod) 


\section{\#\#\#\#CREATING THE DATA SET\#\#\#\# \\ \#\#\#CREATING A POOL OF DISTRIBUTIONS\#\#\#}

\#Here we create a pool of distributions which will be chosen randomly (with

\#replacement) for the GLM analysis\#

Var_Size <- 100

Sample_Size <- 80

Dist_Pool <- c("CHI_SQUARED", "GAMMA", "NORMAL")

\section{\#\#\#PREDICTOR VARIABLES\#\#\#}

\#Creates independent variables from a pool of possible distributions

\#Refer to Dist_Pool for all possible distributions

Y <- rep(0, Sample_Size)

FULL_REGRESSION_RSQUARES $<-\mathrm{c}()$

DATA_COMPLETE <- data.frame $(Y$, matrix (rep(rep(0,Sample_Size),Var_Size $)$, ncol $=$ Var_Size $)$ )

Regressor_Names $<-\mathrm{c}()$

\section{\#\#CREATING THE SIGNIFICANT PREDICTOR VARIABLES\#\#}

$\mathrm{i}<-2$ \#Index of complete data frame

Significant $<-$ round $($ Var_Size*0.20)

while $(i<=$ Significant +1$)\{$ \#We only want the first $20 \%$ of the variables\#

temp_Dist <-sample(Dist_Pool, 1, T) \#Chooses the distribution of predictor variable $\mathrm{Xi}$ at random from the distribution pool

if(temp_Dist == "CHI_SQUARED") \{

df <- sample $(1: 10,1, \mathrm{~T})$ \#Generates data for Chi-Squared distribution

DATA_COMPLETE[,i] <- rchisq(Sample_Size, df)

\#Names respective column in full data frame

Regressor_Names <- c(Regressor_Names, paste("X", i - 1, " CHI-

$\operatorname{SQUARED}(\mathrm{df}=$ ", df, ")", sep = ""))

\} else if(temp_Dist == "GAMMA") \{

alpha $<-$ sample $(1: 10,1, \mathrm{~T})$

beta <- sample $(1: 10,1, \mathrm{~T})$

\#Generates data for Gamma distribution

DATA_COMPLETE[,i] <-rgamma(Sample_Size, alpha, beta)

\#Names respective column in full data frame 


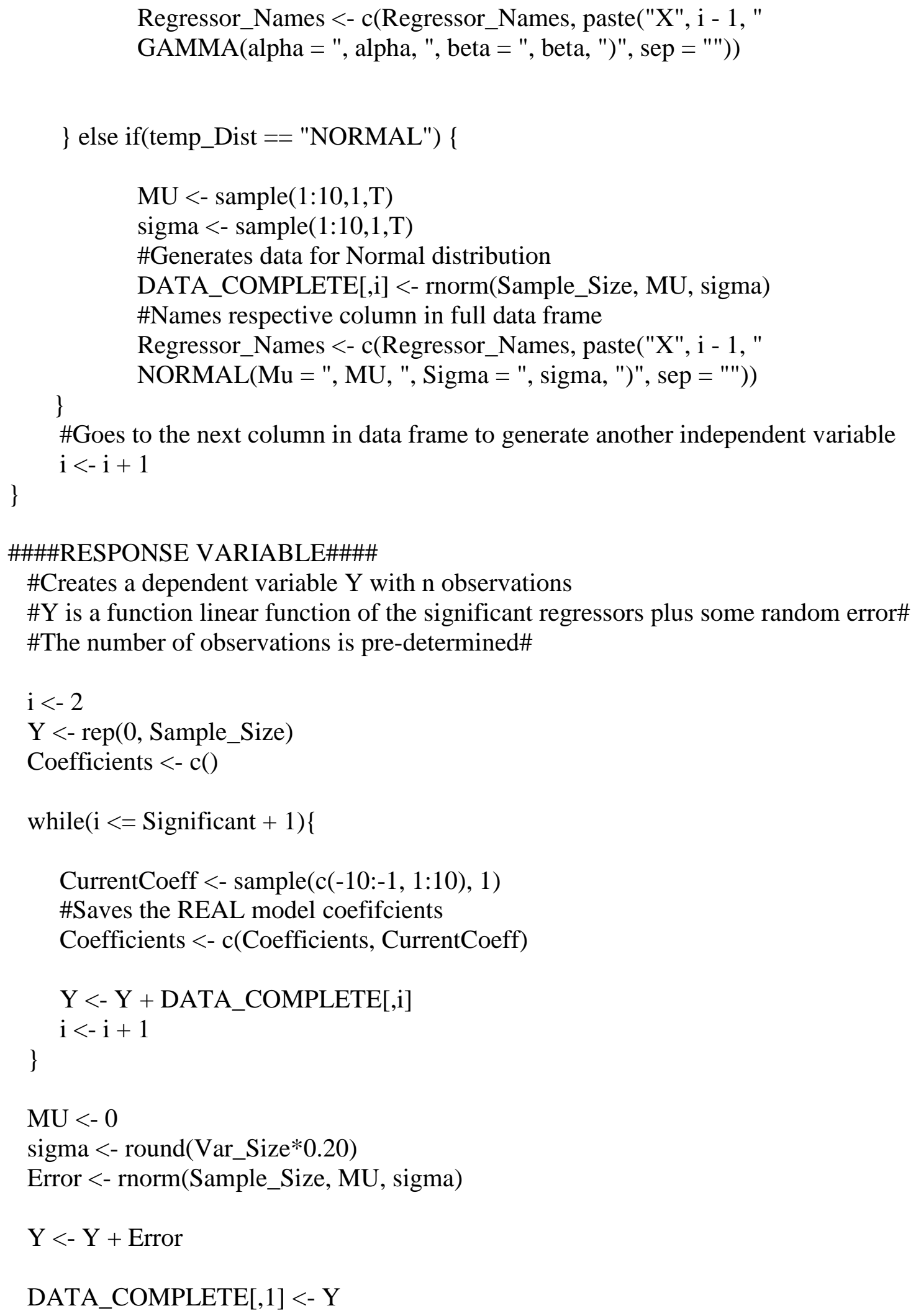




\section{\#\#CREATING THE INSIGNIFICANT PREDICTOR VARIABLES\#\#}

Dist_Pool <- c("POISSON", "BINOMIAL", "CHI_SQUARED", "EXPONENTIAL", "GAMMA", "NORMAL") i <- round(Var_Size*0.20) + 2 \#Index of complete data frame

Significant <- round $($ Var_Size*0.20)

while $(i<=$ ncol(DATA_COMPLETE) \{

\#We only want the first $20 \%$ of the variables\#

temp_Dist <- sample(Dist_Pool, 1, T)

\#Chooses the distribution of predictor variable $\mathrm{Xi}$ at random from the \#distribution pool

if (temp_Dist == "UNIFORM") \{

\#Generates data for Uniform distribution

DATA_COMPLETE[,i] <-runif(Sample_Size)

\#Names respective column in full data frame

Regressor_Names <- c(Regressor_Names, paste("X", i - 1, " UNIFORM", sep = "'))

\} else if(temp_Dist == "POISSON") \{

lambda <- sample $(1: 10,1, \mathrm{~T})$

\#Generates data for Poisson distribution

DATA_COMPLETE[,i] <-rpois(Sample_Size, lambda = lambda)

\#Names respective column in full data frame

Regressor_Names <- c(Regressor_Names, paste("X", i - 1, "

POISSON(lambda = ", lambda, ")", sep = ""))

\} else if(temp_Dist == "BINOMIAL") \{

n $<-$ sample $(1: 10,1, \mathrm{~T})$

$\mathrm{p}<-\operatorname{runif}(1)$

\#Generates data for Binomial distribution

DATA_COMPLETE[,i] <-rbinom(Sample_Size, n, p)

\#Names respective column in full data frame

Regressor_Names <- c(Regressor_Names, paste("X", i - 1, "

BINOMIAL(n = ", n, ", p = ", p, ")", sep = ""))

\} else if(temp_Dist == "BETA") \{ 
alpha $<-$ sample $(1: 10,1, \mathrm{~T})$

beta $<$ - sample $(1: 10,1, \mathrm{~T})$

\#Generates data for Beta distribution

DATA_COMPLETE[,i] <- rbeta(Sample_Size, alpha, beta)

\#Names respective column in full data frame

Regressor_Names <- c(Regressor_Names, paste("X", i - 1, " BETA(alpha

= ", alpha, ", beta = ", beta, ")", sep = ""))

\} else if(temp_Dist == "CHI_SQUARED") \{

df <- sample $(1: 10,1, \mathrm{~T})$

\#Generates data for Chi-Squared distribution

DATA_COMPLETE[,i] <-rchisq(Sample_Size, df)

\#Names respective column in full data frame

Regressor_Names <- c(Regressor_Names, paste("X", i - 1, " CHI-

SQUARED(df = ", df, ")", sep = ""))

\} else if(temp_Dist == "EXPONENTIAL") \{

beta $<-$ sample $(1: 10,1, \mathrm{~T})$

\#Generates data for Exponential dsitribution

DATA_COMPLETE[,i] <- rexp(Sample_Size, beta)

\#Names respective column in full data frame

Regressor_Names <- c(Regressor_Names, paste("X", i - 1, "

EXPONENTIAL(beta = ", beta, ")", sep = ""))

\} else if(temp_Dist == "GAMMA") \{

alpha $<-$ sample $(1: 10,1, \mathrm{~T})$

beta $<$ - sample $(1: 10,1, \mathrm{~T})$

\#Generates data for Gamma distribution

DATA_COMPLETE[,i] <-rgamma(Sample_Size, alpha, beta)

\#Names respective column in full data frame

Regressor_Names <- c(Regressor_Names, paste("X", i - 1, "

GAMMA(alpha = ", alpha, ", beta = ", beta, ")", sep = ""))

\} else if(temp_Dist == "NORMAL") \{

MU <- sample(1:10,1,T)

sigma $<-$ sample $(1: 10,1, \mathrm{~T})$

\#Generates data for Normal distribution 
DATA_COMPLETE[,i] <-rnorm(Sample_Size, MU, sigma)

\#Names respective column in full data frame

Regressor_Names <- c(Regressor_Names, paste("X", i - 1, "

$\operatorname{NORMAL}(\mathrm{Mu}=$ ", MU, ", Sigma = ", sigma, ")", sep = ""))

\}

Coefficients <- c(Coefficients, 0$)$

\#Goes to the next column in data frame to generate another independent variable $\mathrm{i}<-\mathrm{i}+1$

\}

NAMES <- data.frame()

NAMES $<-$ data.frame $($ Regressors $=$ names $($ DATA_COMPLETE[,-1] $)$, Distribution $=$ Regressor_Names, Coefficients $=$ Coefficients)

Corr_Matrix <- data.frame(round(cor(DATA_COMPLETE[,-1]), 3))

\#\#\#\#R-SQUARES OF FULL REGRESSION\#\#\#\#

\#The R-Squared for the FULL regression

\#Full regression includes ALL independent variables

FULL_REG_RSQUARED <- summary $(\operatorname{lm}(\mathrm{Y} \sim$. , data $=$

DATA_COMPLETE))\$r.squared \#Regression on ALL predictor variables

\#\#INDIVIDUAL REGRESSION WITH EACH PREDICTOR VARIABLE\#\#

\#Obtains the individual R-Squared value for each independent variable when \#regressed with the dependent variable

INDIVIDUAL_RSQUARES <- $\mathrm{c}()$ \#Creates an empty list of values for the individual R-Squares

index <- 1 \#Index for the independent variable

\#Obtains each individual R-Squared value and passes it to the list of individual RSquares

while(index $<=$ Var_Size $)\{$

tempRegression $<-\operatorname{lm}(\mathrm{Y} \sim$ DATA_COMPLETE[,index +1 ], data $=$ DATA_COMPLETE) \#Creates temporary regression $\mathrm{Y} \sim \mathrm{Xi}$ 


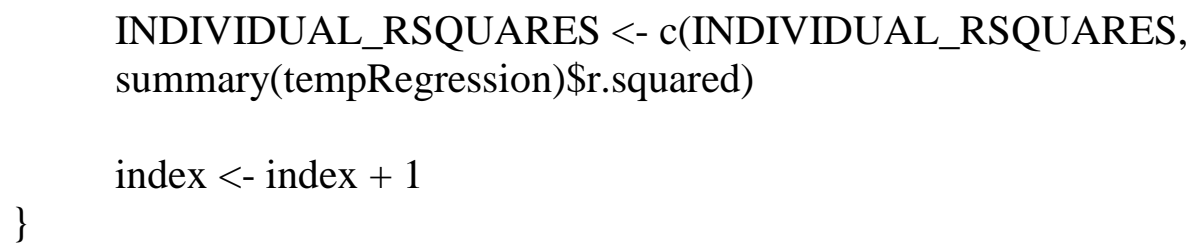




\section{RANDOM_PROBABILITY_POOL}

\section{\#\#\#\#LRP SELECTION\#\#\#\#}

RUNS <- 1000 \#Total number of times we will select regressors based on the created probability pool

CURRENT_RUN <- 1 \#start on first run

REGRESSORS_INFO <- data.frame()

\#The following process will be repeated for each run while(CURRENT_RUN <= RUNS)\{

Prob <- PROBABILITY_POOL \#We need the probability pool for each run

\#\#Choosing Regressors - Probability Pool\#\#

\#Chooses regressors using the probability pool created earlier\#

Ordered_Indexes_Sel <- c() \#Regressor Indexes ordered by selection

Ordered_Names_Sel <- c() \#Regressor Names ordered by selection

Selected_Index $<-\mathrm{c}()$

Selected_Indexes $<-\mathrm{c}()$

index $<-1$

limit <- min $($ Var_Size, Sample_Size/5)

while(index $<=$ limit \{

\#Using Probability Pool\#

picked <- sample(Prob, 1,T) \#The index of the regressor that was picked at random

Prob <- Prob[!Prob \%in\% picked] \#Removes the picked index from the Probability pool

Ordered_Indexes_Sel <- c(Ordered_Indexes_Sel, picked) \#Gets the index of the picked regressor

\#\#UPDATES INDEXES SELECTED UP TO THAT POINT\#\#

if $($ length $($ Selected_Index $)==0)\{$

Selected_Index <- c(as.character(picked))

\}else \{

Selected_Index <- paste0(Selected_Index, ", ", picked)

\} 
Selected_Indexes <- c(Selected_Indexes, Selected_Index $)$

\#Gets the name of the picked regressor

Ordered_Names_Sel <- c(Ordered_Names_Sel, colnames(DATA_COMPLETE)[picked + 1])

index $=$ index +1 \#Goes to the next pick

\}

\#\#DATA_COMPLETE FRAME WITH NAMES AND R-sQUARES\#\#

\#Creates a data frame that contains the regressors in the order by which they were picked along with their simple regression R-Squared and Joint R-Squares\#

index <- 1 \#Start with the first regressor picked

\#Joint R-Squares for each group of regressors using probability pool

Ordered_Joint_RSquares_Sel $<-\mathrm{c}()$

Ordered_Adjusted_RSquares_Sel $<-\mathrm{c}()$

limit <- min $($ Var_Size, Sample_Size/5)

while(index $<=$ limit) \{

\#Based on Probability Pool\#

\#Gets data for Y (1) and selected regressors

tempJointData <- DATA_COMPLETE[,c $(1$,

(Ordered_Indexes_Sel[1:index]) + 1)]

\#NOTE: Regressor index is not the same as the index in the

\#DATA_COMPLETE data frame. Each regressor index is bumbed by one \#unit in the data frame\#

TempJointReg <- $\operatorname{lm}(\mathrm{Y} \sim$, data $=$ tempJointData $)$

\#DATA_COMPLETEndexes_Ordered_Sel only contains regressor

\#indexes so it goes 1 - Var_Size\#

\#Gets the Joint R-Squared for the selected regressors\#

Ordered_Joint_RSquares_Sel <- c(Ordered_Joint_RSquares_Sel,

summary(TempJointReg)\$r.squared)

\#Gets the Joint R-Squared for the selected regressors

Ordered_Adjusted_RSquares_Sel <-c(Ordered_Adjusted_RSquares_Sel, summary(TempJointReg)\$adj.r.squared)

index $<$ - index +1 \#Goes to the next regressor to be added

\}

\#Combines all necessary information into one place\# 
REGRESSORS_INFO_curr <- data.frame $($ Regressor $=$ Ordered_Names_Sel, JointRSquares $=$ Ordered_Joint_RSquares_Sel, AdjustedRSquares $=$ Ordered_Adjusted_RSquares_Sel, Index $=$ Ordered_Indexes_Sel, SelectionOrder $=\operatorname{seq}(1$, limit $)$, Regressors $=$ Selected_Indexes)

\#\#ADDING A COLUMN FOR RUN NUMBER TO R-SQUARES DATA FRAME\#\#

Run <- rep(CURRENT_RUN, limit) \#Run number REGRESSORS_INFO_curr <- dplyr::mutate(REGRESSORS_INFO_curr, Run) \#Adds run number to the combined info

\#\#UPDATING ALL FULL REGRESSION R-SQUARES\#\#

\#Binds this run's information to the general combined information\# REGRESSORS_INFO <- rbind(REGRESSORS_INFO, REGRESSORS_INFO_curr) \#Binds current data frame to big data frame CURRENT_RUN <-CURRENT_RUN + 1 \#Goes to the next run

\}

\#\#\#\#RANDOM SELECTION\#\#\#\#

RUNS <- 1000 \#Total number of times we will select regressors based on the created probability pool

CURRENT_RUN <- 1 \#start on first run

RANDOM_REGRESSORS_INFO <- data.frame()

\#The following process will be repeated for each run while(CURRENT_RUN <= RUNS)\{

\#We need the random probability pool for each run Prob2 <- RANDOM_PROBABILITY_POOL

\#\#Choosing Regressors - Probability Pool\#\#

\#Chooses regressors using the probability pool created earlier\#

Random_Ordered_Indexes_Sel $<-\mathrm{c}()$

Random_Ordered_Names_Sel <- c()

Random_Selected_Index $<-\mathrm{c}()$

Random_Selected_Indexes $<-\mathrm{c}()$

index $<-1$

limit <- min $($ Var_Size, Sample_Size/5) 


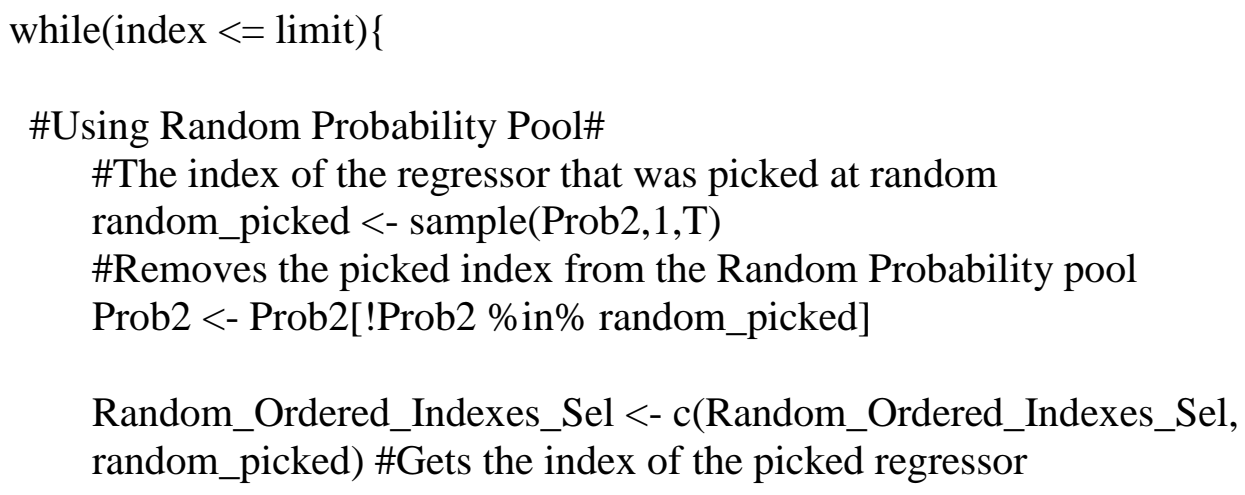

Random_Selected_Indexes <- c(Random_Selected_Indexes, Random_Selected_Index)

\#Gets the name of the picked regressor Random_Ordered_Names_Sel <-c(Random_Ordered_Names_Sel, colnames(DATA_COMPLETE)[random_picked + 1])

index $=$ index +1 \#Goes to the next pick

\}

\#\#DATA_COMPLETE FRAME WITH NAMES AND R-sQUARES\#\#

\#Creates a data frame that contains the regressors in the order by which they were \#picked along with their simple regression R-Squared and Joint R-Squares\#

index <- 1 \#Start with the first regressor picked

\#Joint R-Squares for each group of regressors using probability pool

Ordered_Joint_RSquares_Sel <-c()

\#Joint R-Squares for each group of regressors using random probability pool Random_Ordered_Joint_RSquares_Sel <- c()

\#Joint R-Squares for each group of regressors using random probability pool Random_Ordered_Adjusted_RSquares_Sel <- c () 
limit <- min(Var_Size, Sample_Size/5)

while(index $<=$ limit) \{

\#Based on Ramdom Probability Pool\#

\#Gets data for Y (1) and selected regressors

tempJointData <- DATA_COMPLETE[,c $(1$,

(Random_Ordered_Indexes_Sel[1:index]) + 1)]

\#NOTE: Regressor index is not the same as the index in the DATA_COMPLETE

\#data frame. Each regressor index is bumbed by one unit in the data frame\#

TempJointReg <- $\operatorname{lm}(\mathrm{Y} \sim$., data = tempJointData $)$

\#DATA_COMPLETEndexes_Ordered_Sel only contains regressor indexes so it goes \#1 - Var_Size\#

\#Gets the Joint R-Squared for the selected regressors

Random_Ordered_Joint_RSquares_Sel <-c(Random_Ordered_Joint_RSquares_Sel, summary(TempJointReg)\$r.squared)

Random_Ordered_Adjusted_RSquares_Sel <c(Random_Ordered_Adjusted_RSquares_Sel, summary(TempJointReg)\$adj.r.squared)

index <- index +1 \#Goes to the next regressor to be added

\}

\#Combines all necessary information into one place\#

RANDOM_REGRESSORS_INFO_curr <- data.frame $($ Regressor $=$

Random_Ordered_Names_Sel, JointRSquares =

Random_Ordered_Joint_RSquares_Sel, AdjustedRSquares =

Random_Ordered_Adjusted_RSquares_Sel, Index $=$ Random_Ordered_Indexes_Sel, SelectionOrder $=$ seq(1,limit), Regressors = Random_Selected_Indexes)

\#\#ADDING A COLUMN FOR RUN NUMBER TO R-SQUARES DATA FRAME\#\#

Run <- rep(CURRENT_RUN, limit) \#Run number

RANDOM_REGRESSORS_INFO_curr <dplyr::mutate(RANDOM_REGRESSORS_INFO_curr, Run) \#Adds run number to the random combined info

\#\#UPDATING ALL FULL REGRESSION R-SQUARES\#\#

\#Binds this run's information to the general combined information\#

RANDOM_REGRESSORS_INFO <-rbind(RANDOM_REGRESSORS_INFO, RANDOM_REGRESSORS_INFO_curr) 
CURRENT_RUN <-CURRENT_RUN + 1 \#Goes to the next run

\}

rm(RANDOM_REGRESSORS_INFO_curr, REGRESSORS_INFO_curr, tempJointData)

\#\#\#\#SUMMARY TABLE WITH ALL BEST PROBABLE SUBSETS\#\#\#\#

\#Adjusted R-Squares using BPS\#

BEST_PROBABLE_SUBSETS <- REGRESSORS_INFO

BEST_PROBABLE_SUBSETS <-

BEST_PROBABLE_SUBSETS[order(BEST_PROBABLE_SUBSETS $\$$ JointRSq uares, decreasing $=$ TRUE), ]

BEST_PROBABLE_SUBSETS <- group_by(BEST_PROBABLE_SUBSETS,

SelectionOrder)

BEST_PROBABLE_SUBSETS <- top_n(BEST_PROBABLE_SUBSETS, 1,

JointRSquares)

\# View(BEST_PROBABLE_SUBSETS)

BEST_PROBABLE_SUBSETS <- top_n(BEST_PROBABLE_SUBSETS, 1,

Run)

BEST_PROBABLE_SUBSETS <- ungroup(BEST_PROBABLE_SUBSETS)

BEST_PROBABLE_SUBSETS <-

BEST_PROBABLE_SUBSETS[order(BEST_PROBABLE_SUBSETS $\$$ Selection

Order, decreasing = FALSE), ]

BEST_PROBABLE_SUBSETS <- data.frame $($ SubsetSize =

BEST_PROBABLE_SUBSETS\$SelectionOrder,

Regressors = BEST_PROBABLE_SUBSETS\$Regressors,

RSquares = BEST_PROBABLE_SUBSETS \$JointRSquares,

AdjustedRSquares $=$

BEST_PROBABLE_SUBSETS\$AdjustedRSquares, AdjRSquaresPercentChange $=$

Delt(BEST_PROBABLE_SUBSETS\$AdjustedRSquares)*100)

BEST_PROBABLE_SUBSETS\$RSquares <-

round(BEST_PROBABLE_SUBSETS $\$$ RSquares, 4)

BEST_PROBABLE_SUBSETS\$AdjustedRSquares <-

round(BEST_PROBABLE_SUBSETS\$AdjustedRSquares, 4)

names(BEST_PROBABLE_SUBSETS)[5] <- c("AdjRSquaresPercentChange")

BEST_PROBABLE_SUBSETS[,5] <-

round(BEST_PROBABLE_SUBSETS[,5], 1)

\#\#\#\#DATA FRAMES WITH BEST SUBS FOR ALL SUBSET SEL. METHODS\#\#\#\# \#\#R-Squares Table\#\# 
SQUARES_TABLE $<-$ data.frame $($ SubsetSize $=$

BEST_PROBABLE_SUBSETS\$SubsetSize,

BPS = BEST_PROBABLE_SUBSETS\$RSquares)

\section{\#Best Subset Selection\#}

modelSubset $<-$ regsubsets $(Y \sim$., data $=$ DATA_COMPLETE, nvmax $=$ Var_Size $)$

\#nvmax is the number of variables to consider\#

summarySubset <- summary(modelSubset)

\#Forward Selection\#

modelForward <- regsubsets $(\mathrm{Y} \sim$., data $=$ DATA_COMPLETE, $\mathrm{nvmax}=$ limit, method = "forward") \#nvmax is the number of variables to consider\#

summaryForward <- summary(modelForward)

\#Backward Elimination\#

modelBackward <- regsubsets $(\mathrm{Y} \sim$., data $=$ DATA_COMPLETE, nvmax $=$

Var_Size, method = "backward") \#nvmax is the number of variables to consider\# summaryBackward <- summary(modelBackward)

\#StepWise Regression\#

modelStepwise <- regsubsets $(Y \sim$., data $=$ DATA_COMPLETE, nvmax $=$ Var_Size, method = "seqrep") \#nvmax is the number of variables to consider\# summaryStepwise <- summary(modelStepwise)

RSQUARES_TABLE <- mutate $($ RSQUARES_TABLE, BestSubset $=$ summarySubset\$rsq, Forward = summaryForward\$rsq,

Backward = summaryBackward\$rsq, Stepwise $=$ summaryStepwise $\$$ rsq)

RSQUARES_TABLE[,-1] <-round(RSQUARES_TABLE[,-1], 4)

\#\#Adjusted R-Squares Table\#\#

ADJUSTED_RSQUARES_TABLE $<-$ data.frame $($ SubsetSize $=$ BEST_PROBABLE_SUBSETS\$SubsetSize, BPS $=$ BEST_PROBABLE_SUBSETS $\$$ AdjustedRSquares)

ADJUSTED_RSQUARES_TABLE <mutate(ADJUSTED_RSQUARES_TABLE, BestSubset $=$ summarySubset $\$$ adjr2, Forward = summaryForward $\$$ adjr2,

Backward $=$ summaryBackward\$adjr2, Stepwise $=$ summaryStepwise \$adjr2)

ADJUSTED_RSQUARES_TABLE[,-1] <round(ADJUSTED_RSQUARES_TABLE[,-1],4)

\#\#\#\#PS + BPS MSE\#\#\#\# 
PLOT <- $\operatorname{plot}(\mathrm{x}=$ BEST_PROBABLE_SUBSETS\$SubsetSize, $\mathrm{y}=$

BEST_PROBABLE_SUBSETS\$AdjustedRSquares,

xlab = "Subset Size", ylab = "Adjusted R-Squared")

BPS_Model <- $1 \mathrm{~m}($ Y Apps + Top25perc + P.Undergrad + Outstate +

Room.Board + perc.alumni + Expend, data = DATA_COMPLETE)

BPSModelCoeff <- as.data.frame(summary(BPS_Model)\$coeff)

BPS_MSE <-sum((BPS_Model\$fitted.values -

DATA_COMPLETE $\$$ Y $\left.)^{\wedge} 2\right) /$ Sample_Size

FittedValues <- BPS_Model\$fitted.values

Residuals <- Y - FittedValues

plot(FittedValues, Residuals)

\#\#\#\#RANDOM FORESTS\#\#\#\#

$\mathrm{RF}<-$ randomForest $(\mathrm{Y} \sim$., data $=$ DATA_COMPLETE, ntree $=100$, mtry $=$

Var_Size/3)

RF_DATA <- data.frame $($ Measure = c $($ "Number of Trees", "Splits per Node", "MSE"),

Value $=\mathrm{c}(\mathrm{RF} \$$ ntree, RF\$mtry, sum $($ RF\$predicted -

DATA_COMPLETE $\$$ Y $\left.)^{\wedge} 2\right) /$ Sample_Size)) 